\title{
The mechanism of streak formation in near-wall turbulence
}

\author{
By S. I. CHERNYSHENKO AND M. F. BAIG \\ School of Engineering Sciences, University of Southampton, Southampton, SO17 1BJ, UK
}

(Received 10 July 2003 and in revised form 14 June 2005)

Two conceptual frameworks for the origin of the streaky pattern in near-wall developed turbulent flows are compared. According to the framework that dominated the research over several decades, the pattern of streaks is dictated by the pattern of wall-normal motions via the lift-up mechanism. Various concepts within this framework describe the wall-normal motions as induced by longitudinal vortices, hairpin vortices, vortex packets, etc. According to the newly emerging conceptual framework, the combined action of lift-up of the mean profile, mean shear, and viscous diffusion has its own pattern-forming properties. The pattern of streaks is dictated by these linear effects to a much greater extent than by the pattern of the wall-normal motions. Numerical results supporting the new conceptual framework are presented. An approximate approach for calculating the streak spacing within the new framework is proposed. It is shown to have a significant predictive ability.

\section{Introduction}

\subsection{Streaks}

Observations show the presence of patterns in turbulent flows. Smoke visualization of a turbulent boundary layer can look similar to figure 1( $a$ ) (the picture plane is parallel to the wall). We can clearly see the difference between the horizontal (which is along the mean flow) and vertical (which is parallel to the wall and perpendicular to the mean flow, and which is often called spanwise) directions. The structures shown in figure $1(a)$ are the subject of the present paper. These structures are called streaks, or, more specifically, near-wall streaks.

Experimental measurements and numerical calculations show that from the kinematic viewpoint, streaks are regions of slow fluid motion. In fact, figure 1(a) shows the visualization of the instantaneous longitudinal velocity at the fixed distance from the wall $y^{+}=5.6$, with dark areas corresponding to lower velocity. Superscript + denotes quantities measured in wall units based on the skin friction $\tau$, cinematic viscosity $\nu$, and density $\rho$. In particular, $y^{+}=y_{w} \sqrt{\tau / \rho} / \nu$, where $y_{w}$ is the distance to the wall. The velocity distribution was obtained by direct numerical simulation undertaken in the course of the present study. Streaks are universal, they are always observed in near-wall turbulent flows and their characteristic dimensions are always the same if expressed in wall units and measured immediately at the wall: the length is about 1000 and the spanwise period about 100. To be more accurate, the dimensions depend on the distance to the wall (Smith \& Metzler 1983). As the wall distance increases the streak spacing increases, but streaks become less and less discernible.

Near-wall streaks are very important. Numerical experiments show that if streaks are somehow suppressed, the turbulence intensity is reduced significantly. The mean 
(a)

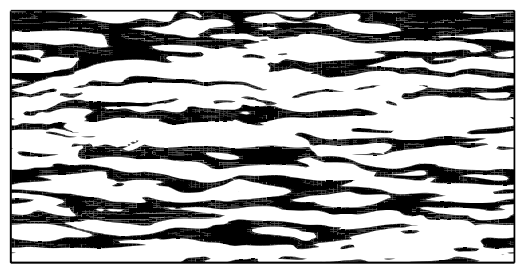

(b)

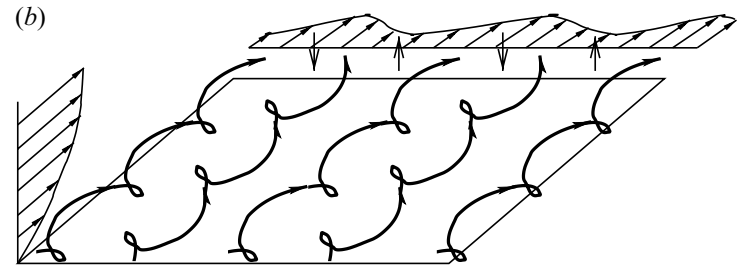

FiguRE 1. (a) Streaks in near-wall turbulent flow at $y^{+}=5.6$ from the wall and $(b)$ the hypothetic mechanism of streak formation by longitudinal vortices.

velocity profile is convex while the streak velocity profiles have inflection points. Convex velocity profiles are much more stable than profiles with inflection points in the sense that perturbations of velocity profiles with inflection points grow much faster. It was also suggested that transient growth of perturbations of the streaky background flow may be of even more importance (Schoppa \& Hussain 2002).

Anyway, it is generally agreed that streaks contribute to the generation of the turbulence energy. Then, the turbulence energy regeneration cycle includes formation of streaks, instability growth at their inflection points or transient growth, and, finally, breakdown of streaks when the perturbations become large. However, there is no generally accepted view on the mechanism of streak formation, and numerous competing theories and conceptual models exist.

In direct numerical simulation of turbulent flow, it is possible to observe the effect of removing specific terms from the Navier-Stokes equations. This is an important method of investigating the mechanism of turbulence (see Jiménez, Pinelli \& Uhlmann 2000). It is known that streaks disappear if the term $v \partial u / \partial y$ is removed, where $u$ and $v$ are the longitudinal and wall-normal velocity components and $y$ is the wall-normal coordinate. Moreover, $u$ can be represented as a sum of the averaged velocity $U$ and a fluctuation, and numerical experiments show (Kim \& Lim 2000) that it is the $v \partial U / \partial y$ term that is responsible for the formation of streaks. In other words, the regions of slow fluid motion appear because there are wall-normal motions advecting the slow-moving near-wall fluid into the region away from the wall.

\subsection{Two conceptual frameworks for streak origin}

For a long time the existence of the pattern of regions of slow longitudinal motion was explained by assuming the existence of a pattern of the wall-normal motion. One of the oldest (Stuart 1965) such patterns is longitudinal vortices as illustrated in figure $1(b)$. Rows of vortices elongated in the direction of the mean flow and rotating in alternating directions capture the fluid and advect it from the wall and to the wall in alternating stripes. The fluid advected from the wall is moving slower than on average at this distance from the wall, while fluid advected to the wall is moving faster. This creates the wavy longitudinal velocity profile at a fixed distance from the wall shown in figure $1(b)$. The same vortices advect smoke if it is released from the wall thus creating streaks similar to figure $1(a)$.

Another well-known candidate for the pattern of wall-normal motions responsible for streaks is a hairpin vortex (see, for example, Smith \& Walker 1994). In this case, the region where the wall-normal velocity is directed away from the wall is not necessarily elongated itself. However, while moving downstream with the hairpin vortex, this region leaves behind a trace in the form of an elongated streak. Other patterns of wall normal motions generating streaks were also proposed. A collection 
(a)

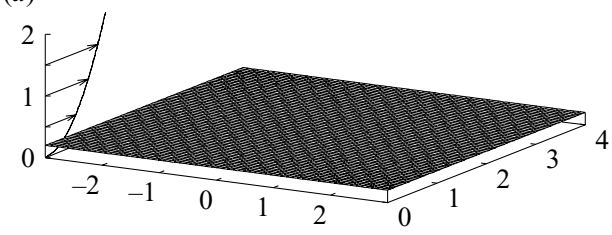

(c)

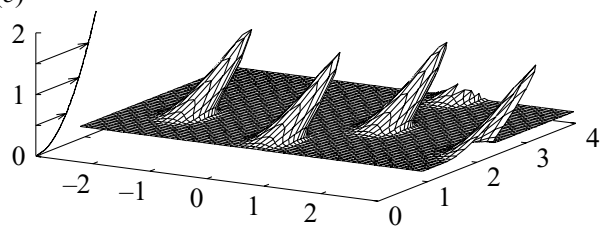

(b)

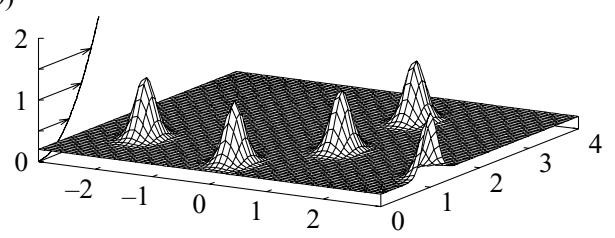

(d)

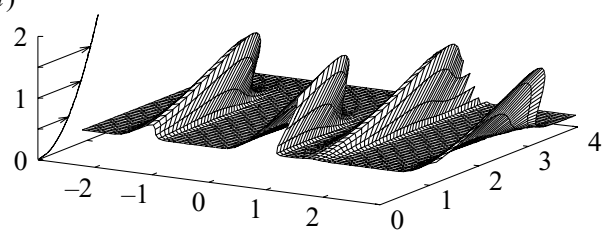

Figure 2. $(a, b)$ Lift-up by isotropic wall-normal motions, $(c)$ effect of mean shear and (a) diffusion.

of relevant papers can be found in Panton (1997). Other references are Jang, Benney \& Gran (1986); Sreenivasan (1988); Brooke \& Hanratty (1993); Butler \& Farrell (1993); Waleffe, Kim \& Hamilton (1993); Hamilton, Kim \& Waleffe (1995); Nikitin \& Chernyshenko (1997); Schoppa \& Hussain (1998); Waleffe (2003), and even this list is probably incomplete.

While streaks are easy to observe in experiment or direct numerical simulations, observing the corresponding pattern of wall-normal motions is not so easy. This may explain why, even after several decades of research, there is no general agreement on which pattern of wall-normal motions is responsible for streaks. It appears, however, that the idea that the pattern of streaks is dictated by the pattern of wall-normal motions is generally accepted by a large number of workers. For brevity, we will call this idea the first conceptual framework of streak formation.

There is an alternative to that idea. Consider the case when the wall-normal velocity has no pattern at all, so that it is isotropic with respect to directions parallel to the wall. Figures 2(a) and 2(b) illustrate the effect of lift-up by such wall-normal motions. As was mentioned, streaks of a passive scalar (such as smoke) and the velocity streaks are likely to be created by the same mechanism, and we will describe the process in terms of a passive scalar. In figure 2, the surface indicates the boundary of the region of high concentration of a passive scalar released from the wall. Initially, figure 2(a), the boundary is assumed to be flat, but the lift-up by wall-normal motion will deform it, figure $2(b)$. Imagine now a visualization plane parallel to the wall at the distance from the wall that is somewhat greater than the distance to the initial position of the boundary. The visualization plane will cut through the surface shown in figure $2(b)$. Visualization then can be performed by painting with colour the regions of the visualization plane where the scalar concentration is high, that is those parts of the visualization plane that are beneath the surface plotted in figure $2(b)$. The resulting picture, however, will not have a streaky pattern because the wall-normal motions are assumed to have no pattern. In other words, lift-up by isotropic wall-normal motions does not create streaks.

Consider now how this process is affected by the mean shear. The shear will tilt and stretch the lifted volumes, as shown in figure $2(c)$. In the visualization plane, the picture will only be shifted downstream, so that no streaks will appear. However, 
tilting and stretching will lead to an increase of the wall-normal gradients. The effect of the wall-normal diffusion is shown in figure $2(d)$. Now the visualization will show a streaky pattern in the visualization plane.

In this case, the origin of the streaky pattern is not in the pattern of the wallnormal motion, but in the pattern-forming properties of the combined action of lift-up, shear and diffusion. Note that the described mechanism of streak formation by non-structured wall-normal motions is not unique. Similarly to longitudinal vortices, hairpins and so on being the various alternatives within the first conceptual framework of streak formation, various mechanisms can be proposed within the second conceptual framework. Note also that these two conceptual frameworks are not exclusive: the characteristics of streaks in real turbulent flows are naturally determined by both the pattern of wall-normal motion and the pattern-forming properties of the combined action of lift-up, shear and diffusion. In the present paper, we will investigate which of them dominates.

For explaining streaks within the second framework, it is sufficient to consider only diffusion, lift-up of the mean profile and mean shear. These effects are governed by the linearized Navier-Stokes equations. The ability of the linearized Navier-Stokes equations to predict streaky patterns is well known. In particular, Lee, Kim \& Moin (1990) compared the results of direct numerical simulations with the predictions of the rapid distortion theory, which is based on linearized equations, and concluded that, 'the dominant mechanism in the production (and maintenance) of the preferred structures (i.e. streaks) in all turbulent shear flows at high shear rate is a selective amplification of eddies primarily by the linear interaction with mean shear'. Landahl (1989) modelled the near-wall turbulent flow field as the linear response of the mean shear flow to random forcing and obtained a good qualitative agreement for the evolution of coherent structures educed by the VITA conditional sampling technique. Butler \& Farrell (1993) calculated the optimal perturbations of the flow in a plane channel linearized about the turbulent mean velocity. Optimal perturbations predicted streaks, but in order to obtain streak spacing agreeing with experiment, Butler \& Farrell had to limit the lifetime of the perturbation by the eddy turnover time. Carpenter et al. (2003) looked for the strongest response of the boundary layer to a small streamwise vorticity source and obtained a satisfactory agreement with experiment for streak spacing both for rigid and compliant walls. However, these observations did not lead to a wide acceptance of the second framework, maybe because of the need for a clear and logical explanation of how linearized equations can give predictions about the essentially nonlinear phenomenon of turbulence.

\subsection{A simple model illustration}

The difference between the two frameworks can be illustrated further with a simple model. This model will also be used throughout the paper for explaining the ideas of the numerical experiments and of the theoretical approach. Let periodic functions $\tilde{u}(z)=\sum_{n} \tilde{u}_{n} \exp (\mathrm{inz} z)$ and $\tilde{f}(z)=\sum_{n} \tilde{f}_{n} \exp (\mathrm{in} z)$ be governed by the system of equations

$$
\tilde{u}=\mathrm{L} \tilde{f}, \quad \tilde{f}=\mathrm{N}[\tilde{u}],
$$

where $\mathrm{L}$ is a linear operator such that

$$
\tilde{u}_{n}=\tilde{\lambda}_{n} \tilde{f}_{n},
$$

and $\mathrm{N}$ is a nonlinear operator, so that the whole system is nonlinear. Suppose that the solution of this system has a pattern in the sense that $\tilde{u}(z)$ is close to 

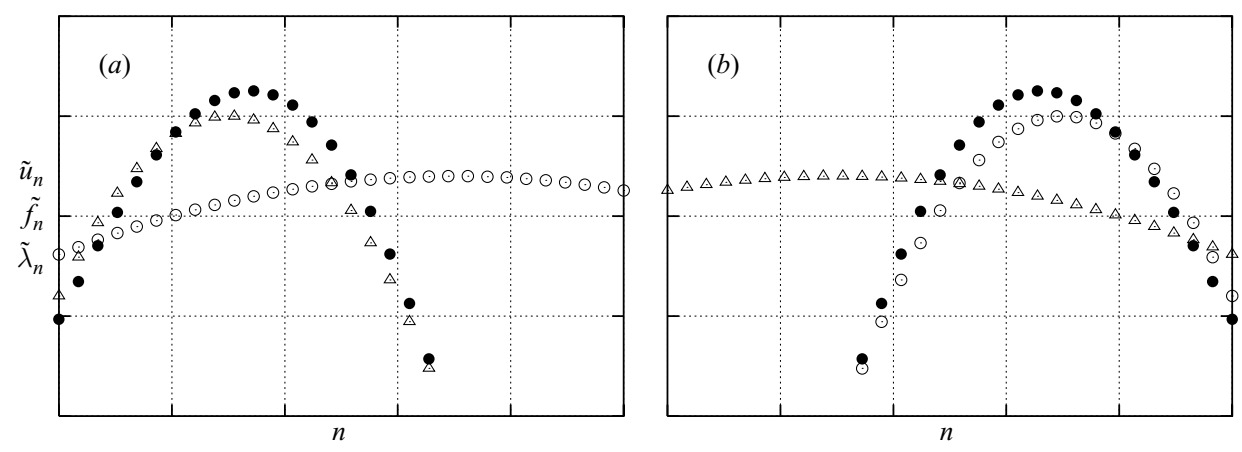

FIGURE 3. A model for the two frameworks: $\bullet, \tilde{u}_{n} ; \triangle, \tilde{f}_{n} ; \bigcirc, \tilde{\lambda}_{n} ;(a)$ case 1 and $(b)$ case 2.

sinusoidal, $\tilde{u}(z) \approx \exp (\mathrm{i} k z)$, with a certain $k$. We may consider $\tilde{u}(z)$ as a model for longitudinal velocity having a streaky pattern, and $\tilde{f}(z)$ is whatever forces the streaks. In the simplest case, $\tilde{f}(z)$ may be considered as a model of the wall-normal velocity component, but actually in the studies based on the linearized Navier-Stokes equations, and also in the present paper, the forcing usually represents the nonlinear terms of the Navier-Stokes equations rewritten in fluctuations as dependent variables.

The dominance of a specific wavelength in $\tilde{u}(z)$ means that the plot of $\left|\tilde{u}_{n}\right|$ versus $n$ has a pronounced maximum at $n=k$. In view of (1.2), the two simplest explanations of the existence of this maximum are as follows.

Case 1. There is a pronounced maximum of $\tilde{f}_{n}$ near $n=k$ while the plot of $\tilde{\lambda}_{n}$ is relatively flat (figure $3 a$ ). In this case, the pattern of $\tilde{u}(z)$ ('streaks') is dictated by the pattern of $\tilde{f}(z)$ ('wall-normal motions'). This case corresponds to the first conceptual framework.

Case 2. There is a pronounced maximum of $\tilde{\lambda}_{n}$ near $n=k$ while the plot of $\tilde{f}_{n}$ is relatively flat (figure $3 b$ ). In this case, the pattern of $\tilde{u}(z)$ ('streaks') is dictated by the pattern-forming properties of L ('combined action of lift-up of the mean profile, mean shear and diffusion'). This case corresponds to the second conceptual framework.

\subsection{The structure of the paper}

The first results obtained within this study were purely theoretical. The numerical calculations were performed later, and the illustrative model of $\S 1.3$ is the latest addition. However, in this paper they are presented in a more logical order. The efforts to disseminate these results showed that the main difficulty in understanding them is due to confusing the two conceptual frameworks, such as, for example, misinterpreting certain velocity fields arising in the theoretical solutions within the second framework as a model of the pattern of wall-normal motions dictating the pattern of streaks within the first framework. The illustrative model of $\S 1.3$ is used throughout the paper in order to help to avoid the confusion. The elements of this model are distinguished with tildes. The structure of the paper is also aimed at clarifying the conceptual issues first. Section 2 describes the numerical experiments specially designed to determine which of the two conceptual models corresponds better to the real turbulent flow. Section 3 describes an approximate approach to predicting streak spacing within the second conceptual framework. Section 4 is devoted to comparisons, and $\S 5$ contains the discussion and conclusions. More technical material is given in the Appendices. 


\section{Numerical tests}

\subsection{Scalar streaks created by unstructured wall-normal motions}

We will now design numerical experiments aimed at determining which of the frameworks describes better the streak formation mechanism in real turbulent flow. The model (1.1) suggests two such experiments, if only (1.1a), can be isolated. We can then replace $\tilde{f}$ obtained from the solution of (1.1) with some unstructured function $\tilde{g}(z)$ such that its Fourier coefficients $\tilde{g}_{n}$ do not have a pronounced maximum, and analyse the behaviour of $\mathrm{L} \tilde{g}$. In case 1, illustrated by figure $3(a)$ and corresponding to the first framework, $L \tilde{g}$ will have no pronounced pattern, while in case 2 the pattern of $\mathrm{L} \tilde{g}$ will be similar to the pattern of $\tilde{u}$.

In real flows, isolating longitudinal and wall-normal motions is not so easy because they are linked by the continuity equation. However, since passive scalars also exhibit streaks, the numerical experiment can be performed with a passive scalar.

We will later consider flows with several passive scalars present at the same time. For simplicity, we assume that the Schmidt number for each scalar is equal to unity. Then a passive scalar $\theta_{i}$ is governed by the equation

$$
\frac{\partial \theta_{i}}{\partial t}+\boldsymbol{u} \cdot \nabla \theta_{i}=S_{i}(y)+\frac{1}{R e} \nabla^{2} \theta_{i},
$$

where $S_{i}(y)$ is the source term. We have modified the pseudospectral plane channel code of Sandham \& Howard (1998) in order to solve simultaneously the NavierStokes equations and an arbitrary number of passive-scalar equations. All calculations were performed at $R e=360$ in a computational box of size $L_{x} \times L_{y} \times L_{z}=6 \times 2 \times 3$ using $64 \times 64 \times 160$ modes (Chebyshev in wall-normal and complex-valued Fourier in homogeneous directions). Constant values were prescribed at the channel walls as the boundary conditions for the scalars.

Prescribing different $S_{i}(y)$, we can obtain different mean profiles for $\theta_{i}$. In particular, it is possible to adjust the source term in such a way that the mean profile of the scalar will coincide with the mean profile of the longitudinal velocity. Then the equation for the passive-scalar fluctuation will coincide with the equation for the fluctuation of the longitudinal velocity apart from the term with a longitudinal derivative of the pressure fluctuation. Our calculation confirmed that this term has almost no effect on streaks; the instantaneous pictures of the streaks of this scalar and the velocity streaks almost coincided. This was expected because streaks are elongated and hence the longitudinal gradients are of little importance and because this similarity is well known from the numerical simulations of Kim \& Moin (1989) and Kasagi, Tomita \& Kuroda (1992). This feature confirms also that the mechanisms of formation of scalar streaks and velocity streaks are the same.

Naturally, in this calculation the wall-normal motions creating scalar streaks possess all the typical patterns of wall-normal motions in a turbulent flow, since these motions were obtained from simultaneously running direct numerical simulation of turbulent flow in a channel. In a separate calculation these motions were replaced with artificially synthesized unstructured motions. More specifically, we solved numerically the equation

$$
\frac{\partial \theta}{\partial t}+\left(U \boldsymbol{e}_{x}+\nabla \phi\right) \cdot \nabla \theta=S(y)+\frac{1}{R e} \nabla^{2} \theta,
$$

using the same boundary conditions as for (2.1). The source term was adjusted to ensure that the mean scalar profile again coincided with the mean velocity profile 
(a)

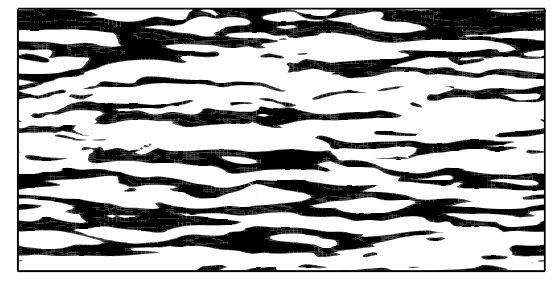

(b)

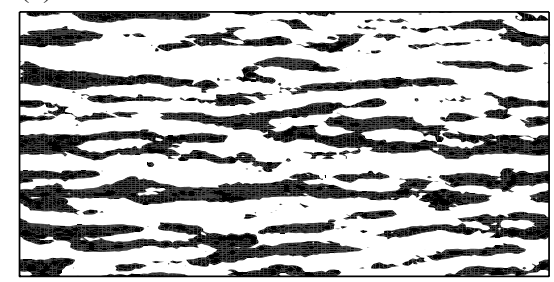

Figure 4. (a) Velocity streaks in near-wall turbulent flow at $y^{+}=5.6$ from the wall and (b) passive scalar streaks in the flow with unstructured potential perturbations at the same distance from the wall.

in the turbulent flow, $\langle\theta\rangle=\Theta(y)=\langle u\rangle=U(y)$. Here and throughout the paper angle brackets $\langle$.$\rangle denote averaging.$

The fluctuation potential satisfied the Laplace equation and the impermeability condition at the lower wall $y=-1$ (the upper wall is at $y=1$ ) and was given by the formula

$$
\begin{aligned}
\phi= & 4 \times 10^{-4} \operatorname{Re} \sum_{k=-16}^{k=+16} \sum_{l=-16}^{l=+16} P_{k l}(t) \exp \left(\mathrm{i}\left(2 \pi \alpha_{k l}(t)+k_{x} x+k_{z} z\right)\right) \\
& \times\left(\exp \left(\sqrt{k_{x}^{2}+k_{z}^{2}}(y-1)\right)+\exp \left(-\sqrt{k_{x}^{2}+k_{z}^{2}}(y+3)\right)\right),
\end{aligned}
$$

where $k_{x}=2 \pi k / L_{x}$ and $k_{z}=2 \pi l / L_{z}$ with $L_{x}=6$ and $L_{z}=3$.

The coefficients $P_{k l}(t)$ and $\alpha_{k l}(t)$ were generated at discrete time instants separated by a time interval $\Delta t_{\text {ran }}=0.05$ as independent random quantities uniformly distributed in $(0,1)$ and $(-0.5,0.5)$, respectively, and spline-interpolated in between. The numerical calculation itself used a much smaller time step which ensured sufficient time resolution.

By construction, in the second calculation, the velocity field had constant vorticity $\boldsymbol{\omega}=-U^{\prime}(y) \boldsymbol{e}_{z}$, that is, it did not contain any vortical structures. Moreover, this velocity field was isotropic in planes parallel to the wall. However, it generated scalar streaks. These scalar streaks are shown in figure 4 side-by-side with the velocity streaks of the turbulent flow. Because of the limited computational resources, only a few more calculations were performed with different $\Delta t$ and $\phi$. This was sufficient to ensure that creation of streaks by unstructured wall-normal motion is a robust phenomenon. More calculations would be required to verify that the agreement in streak spacing is due to the mean scalar profile having the same shape as the mean turbulent velocity profile rather than being a coincidence.

The main point of this numerical experiment is clear: removing the pattern of wallnormal motions does not remove streaks. This behaviour is similar to the behaviour of the simple model in case 2 , but not in case 1 . Therefore, the results of this experiment are in favour of the second conceptual framework.

\subsection{Variation of streak parameters with the mean profile}

Another experiment suggested by the model (1.1) consists of the following. Rather than replacing $\tilde{f}_{n}$ in $\tilde{u}_{n}=\tilde{\lambda}_{n} \tilde{f}_{n}$ with a set of values without a pronounced maximum, we can try to replace $\tilde{\lambda}_{n}$ with such a set. However, it is not obvious how to design a similar numerical experiment with the Navier-Stokes equations or the passive-scalar equation. The variation of this would be to modify $\tilde{\lambda}_{n}$ so that the maximum of 
$\left|\tilde{\lambda}_{n}\right|$ is shifted. Then, in case 1 the modified $\mathrm{L}_{\text {mod }} \tilde{f}$ would have the same dominant wavelength, since it is dictated by the dominant wavelength of $\tilde{f}$, while in case 2 its dominant wavelength would be shifted too.

The method of modifying the linearized operator in turbulent flow with a passive scalar is suggested by the physical mechanism described in $\S 1$. This mechanism involves the lift-up of the mean profile of the passive scalar. Therefore, modifying $S_{i}(y)$ in (2.1) will affect the linear mechanism. At the same time, since the scalar is passive, the velocity field will not be affected and will, therefore, possess whatever patterns can be thought of as dictating streaks. We performed numerical calculations of the turbulent flow in a plane channel with several scalars having different mean profiles. Other parameters were the same as in $\S 2.1$. The larger the variation in streaky pattern, the more conclusive is the result of such an experiment. Also, large variation in the patterns extends the scope of the comparison for theoretical predictions of streak spacing. The approach used for selecting the mean profiles of the passive scalars is described in Appendix A. Results of this numerical experiment showed that the scalars with different mean profiles simultaneously present in turbulent flow can have streak spacing varying by an order of magnitude. These data are presented in more detail in $\S 4.3$ where they are compared with theoretical predictions.

Since the pattern of wall-normal motions was the same for all scalars, the large variation of streak spacing caused by the variation in the mean profiles indicates that the results of the second numerical experiment also are in favour of the second conceptual framework.

The third, and quite obvious, test for deciding which framework is more appropriate for real turbulent flow is to compare the characteristic spanwise scale of longitudinal velocity with the spanwise scale of the wall-normal velocity in the same flow. All the necessary data are available in various direct numerical simulation databases, and the result is in fact known: in most cases these scales differ by a factor of about two, see, for example, Chernyshenko \& Baig (2005), which outlines the material presented at the London Mathematical Society Sponsored Workshop on New Developments and Applications in Rapid Fluid Flow, Durham, UK, 2003. Since such a comparison does not distinguish the spanwise scales of elongated and not elongated patterns, it is of limited significance, but nevertheless it fits better into the second framework than into the first.

In general, all three tests favour the second conceptual framework. Therefore, it is reasonable to investigate the possibility of developing a predictive tool based on the second framework. This is the objective of the next section.

\section{Generalized optimal perturbations}

\subsection{The idea}

In case 1 of the simple model of $\S 1.3$, predicting the dominant wavelength of $\tilde{u}$ would require the prediction of the dominant wavelength of $\tilde{f}$. This, in turn, would require the analysis of at least the nonlinear operator $\mathrm{N}$ or even the analysis of the entire system (1.1). In case 2, predicting the dominant wavelength requires the prediction of the wavenumber $n$ of the largest $\tilde{\lambda}_{n}$. This can be done by analysing only the linear operator L, which should be easier than analysing the nonlinear operator. Determining which wavelength is most favoured by a linear operator can be done in different ways. For example, we can look for the maximum of $\|\mathrm{L} \tilde{w}(z)\|^{2}=\sum_{n} \tilde{\lambda}_{n}^{2} \tilde{w}_{n}^{2}$ 
over all possible $\tilde{w}(z)$ such that $\|\tilde{w}(z)\|=1$. Obviously, the maximum is attained at $\tilde{w}(z)=\tilde{f}_{\text {opt }}(z)=\exp (k \mathrm{i} z)$, where $k$ is such that $\left|\tilde{\lambda}_{k}\right|$ is at the maximum.

To apply the same idea to turbulent flow, the full Navier-Stokes equations can be rewritten in fluctuations $\boldsymbol{u}^{\prime}=\boldsymbol{u}-\boldsymbol{U}, p^{\prime}=p-P$ in the following form,

$$
\begin{gathered}
\overbrace{\frac{\partial \boldsymbol{u}^{\prime}}{\partial t}+\boldsymbol{U} \cdot \nabla \boldsymbol{u}^{\prime}+\boldsymbol{u}^{\prime} \cdot \nabla \boldsymbol{U}+\nabla p^{\prime}-\frac{1}{R e} \nabla^{2} \boldsymbol{u}^{\prime}}^{\text {linear mechanism }\left(\sim \mathrm{L}^{-1} \text { in the model }\right)}=\boldsymbol{F}, \\
\quad \overbrace{(\sim \mathrm{N} \text { in the model })}^{\boldsymbol{F}=-\boldsymbol{u}^{\prime} \cdot \nabla \boldsymbol{u}^{\prime}-\boldsymbol{U} \cdot \nabla \boldsymbol{U}-\nabla P-\frac{1}{R e} \nabla^{2} \boldsymbol{U},},
\end{gathered}
$$

$$
\nabla \boldsymbol{u}^{\prime}=0
$$

Then, setting aside (3.2), equations (3.1) and (3.3) can be considered as a forced response problem for the linearized Navier-Stokes equations, and the pattern most favoured by these equations can be sought by maximizing $\left\|\boldsymbol{u}^{\prime}\right\|$ over all $\boldsymbol{F}$ such that $\|\boldsymbol{F}\|=1$. It appears that so far this program has not been implemented in full. However, if the set of functions $\boldsymbol{F}(t, x, y, z)$ over which the maximization is performed is limited to functions of the form $\boldsymbol{F}=\delta(t) \boldsymbol{u}_{0}(x, y, z)$, where $\delta(t)$ is the Dirac delta, then with a suitable choice of the norms, this problem coincides with the so-called optimal perturbation problem. Limiting $\boldsymbol{F}$ to such functions can be considered as a simplification aimed at obtaining an approximate solution.

\subsection{Butler \& Farrell approach}

The linearized Navier-Stokes equations are the natural tool for investigating the transition to turbulence. Optimal perturbations are used to describe the bypass transition scenario. The optimal perturbation is the perturbation whose energy grows by a maximum factor prior to decay. The exact formulation of the optimal perturbation problem is: find the initial condition $\boldsymbol{u}_{\mid t=0}^{\prime}=\boldsymbol{u}_{0}^{\prime}$ and the corresponding solution $\boldsymbol{u}^{\prime}$ of (3.1), (3.3) (with $\boldsymbol{F}=0$ ) so that $\left\|\boldsymbol{u}_{0}^{\prime}\right\|=1$ and the $\max _{t \rightarrow 0}\left\|\boldsymbol{u}^{\prime}\right\|$ is the greatest possible. Here, $\|$.$\| denotes the standard energy norm. There exists extensive literature$ on the subject (see Butler \& Farrell 1992; Trefethen et al. 1993; Criminale, Jackson \& Lasseigne 1995, and references therein). With $\boldsymbol{U}$ taken as the velocity of the laminar flow, the solution of this problem shows that in practically interesting cases the energy of the initial perturbation can grow by a factor of several hundred or even more. Naturally, this is important for the transition to turbulence.

The interpretation given in $\S 3.1$ is, of course, different from that accepted in the stability analysis. In $\S 3.1$, the perturbations are not even assumed small, it is implied only that the mean value of the perturbation is zero. Butler \& Farrell (1993) assumed $\boldsymbol{U}$ to be the mean velocity in turbulent flow in a plane channel and calculated the optimal perturbations for the Reynolds number based on the dynamic velocity and the channel half-width $R e=180$ (this corresponds to the Reynolds number based on the maximal velocity and the channel width $R e_{\max } \approx 6480$ ). The structure of the optimal perturbation involved strong streaks. The perturbation energy grew significantly (by a factor of 235), but the streak spacing was much greater $\left(l^{+}=540\right)$ than in reality (at the wall $l^{+} \approx 100$ ). The time required for the energy to attain the maximum value turned out to be much greater that the characteristic time scale of small-scale turbulent fluctuations. Then they suggested an assumption that in reality the perturbation exists only over a limited time equal to the so-called eddy turnover 
time $t_{e}=q^{2} / \epsilon$, which is the ratio of the characteristic turbulent velocity, defined as $q^{2}=\left\langle u_{i} u_{i}\right\rangle$, to the dissipation rate, $\epsilon=\left\langle v u_{i, j} u_{i, j}\right\rangle$. After this time, the perturbation is assumed to be destroyed by turbulent fluctuations. The eddy turnover time depends on the distance to the wall, and Butler \& Farrell (1993) proposed to take its value at the centre of the streak. With this adjustment, the streak spacing was found to be close to the experiment. However, Waleffe \& Kim (1997) pointed out that this adjustment simply transforms the length-scale selection problem into the time-scale selection problem.

Anyway, the above argument leading to limiting the evolution time of the optimal perturbation by the eddy turnover time is far from perfect. It implies that the optimal perturbation remains a good approximation for the turbulent flow field for at least the eddy turnover time, that is, that almost every time a streak is about to appear, the fluctuating velocity of the real flow happens to be close to the initial condition for the optimal perturbation and that after that, $\boldsymbol{F}$ in (3.1)-(3.3) remains negligible for a period equal to the eddy turnover time. This assumption is unrealistic. The $\S 3.1$ interpretation shows also that this assumption is unnecessary. This can be illustrated more clearly by including one more function in the illustrative model of $\S 1.3$. Let $\tilde{c}(z)$ be governed by an additional equation

$$
\tilde{c}(z)=\mathrm{M} \tilde{f}(z),
$$

where the operator $\mathrm{M}$ is linear and such that $\tilde{c}_{n}=\tilde{\mu}_{n} \tilde{f}_{n}$. If $\left|\tilde{\mu}_{n}\right|$ has a pronounced maximum at $n=l$, then not only $\tilde{u}(z)$ but also $\tilde{c}(z)$ will be nearly sinusoidal, but with $l \neq k$ their dominant wavelengths are different. The dominant wavelength of $\tilde{c}(z)$ can be found approximately by maximizing $\|\mathrm{M} \tilde{w}(z)\|$ over all $\tilde{w}(z),\|\tilde{w}(z)\|=1$. The maximum is attained at $\tilde{w}(z)=\tilde{f}_{\mathrm{opt}}^{c}(z)=\exp (l \mathrm{i} z)$, thus giving the period of $\tilde{c}(z)$. Therefore, for the same $\tilde{f}(z)$, we have two different optimal perturbations indicating the different Fourier modes of $\tilde{f}(z)$ that are the most efficient in inducing the corresponding Fourier modes of $\tilde{u}(z)$ and $\tilde{c}(z)$. Note that if the system $(1.1 a, b),(3.4)$ were of the case 1 type (that is if the first conceptual framework was more appropriate for it), then both $\tilde{u}(z)$ and $\tilde{c}(z)$ would have the same dominant wavelength close to the dominant wavelength of $\tilde{f}(z)$.

While the solution $\tilde{f}(z), \tilde{u}(z), \tilde{c}(z)$ and the 'optimal perturbation' $\tilde{f}_{\text {opt }}(z), \tilde{u}_{\text {opt }}(z)=$ $L \tilde{f}_{\text {opt }}(z)$ have similar patterns of $\tilde{u}(z)$ and $\tilde{u}_{\text {opt }}(z)$ (one has a dominant wavelength and the other is strictly sinusoidal with approximately the same wavelength), the pattern of $\tilde{f}(z)$ can be quite different from the pattern of $\tilde{f}_{\text {opt }}(z)$. In fact, $\tilde{f}_{\text {opt }}(z)$ gives no information about $f(z)$. The same applies to $\tilde{f}_{\text {opt }}^{c}(z)$. Therefore, in the context of developed turbulent flows $\uparrow$ optimal perturbations should not be interpreted as approximations of the real solution. They are only a tool for investigating the properties of the linearized Navier-Stokes equations, including those properties which dictate the patterns observed in turbulent flows. Butler \& Farrell (1993) did not explain why the streak spacing of the optimal perturbation they calculated should be close to the streak spacing in the real turbulent flow. Their explanation has to be different from the explanation presented here because if, as in our explanation, the optimal perturbation is only a tool for investigating the relevant property of the linearized

$\dagger$ In the stability analysis we can argue that occasionally small-amplitude perturbations can coincide with the initial condition of the optimal perturbation and remain close to it while experiencing the transient growth. However, at least part of the reasoning presented here can also be transferred to the interpretation of optimal perturbation in the stability analysis. 
Navier-Stokes equation and not an approximation of the real flow, then it cannot be destroyed by turbulent perturbation, and introducing the eddy turnover time adjustment cannot be justified.

\subsection{Specific formulation used in the present study}

On the other hand, there is another explanation why, without the eddy-turnover-time adjustment, the standard optimal perturbation theory does not predict the correct streak spacing. The model illustration of $\S 3.2$ makes it especially clear by providing two different optimal perturbations for the same system. A convenient way of dealing with this is to consider $\|\tilde{u}\|$ and $\|\tilde{c}\|$ as different norms of the same solution $(\tilde{u}, \tilde{c}, \tilde{f})$, say, $\|(\tilde{u}, \tilde{c}, \tilde{f})\|_{1}=\|\tilde{u}\|$ and $\|(\tilde{u}, \tilde{c}, \tilde{f})\|_{2}=\|\tilde{c}\|$. (Strictly speaking these norms apply to different functional spaces owing to the difference between the zero elements of these spaces. However, this is of no importance in the context of the present study.) Obviously, predicting different features of the solution requires maximisation of different norms.

It is well known that streaks are essentially a near-wall feature. The streak spacing increases and streak strength decreases with the distance from the wall. The energy norm used by Butler \& Farrell is equivalent to some kind of averaging across the channel. A solution with very strong streaks localized near the walls and very weak in other parts of the flow domain can have quite a small energy norm as compared to a solution of moderate amplitude but occupying a considerable part of the flow domain. Therefore, this norm favours large-scale solutions and may ignore solutions with the strongest streaks. In fact, experimental and direct numerical simulation data give different values for streak spacing in different visualization planes, so that the streak spacing is a function of the distance to the wall. The logic then requires that the optimal perturbation calculated for predicting streak spacing in a specific visualization plane should be obtained by maximizing a measure of streak strength within this visualization plane.

For a plane channel flow, the most natural choice is

$$
\|\boldsymbol{u}\|_{y}^{2}=\lim _{a, b \rightarrow \infty} \frac{1}{a b} \int_{x, z=0}^{x=a, z=b} u^{2}(x, y, z) \mathrm{d} x \mathrm{~d} z .
$$

Here, the longitudinal, wall-normal and spanwise coordinates and perturbation velocity components are denoted $x, y, z$ and $u, v, w$, respectively, so that $\boldsymbol{u}=(u, v, w)$. In contrast to other theories, this approach gives the streak spacing not as a single value but as a function of the distance $\left|y-y_{\text {wall }}\right|$ to the wall.

The norm (3.5) cannot be used for imposing the restriction on the possible initial distribution, since it does not limit the cross-flow component. It also does not limit the longitudinal velocity at other $y$. Hence, it would not limit the maximum growth. Therefore, another norm has to be used for this purpose. Accordingly, we formulate a generalized optimal perturbation problem: to find $\boldsymbol{u}_{0}^{\prime}$ at which the solution norm $\left\|\boldsymbol{u}^{\prime}\right\|_{s}$ reaches its maximum over all $t>0$ and over all possible $\boldsymbol{u}_{0}^{\prime}$ such that the initial condition norm $\left\|\boldsymbol{u}_{0}^{\prime}\right\|_{i}=1$. Alternative formulation is to find

$$
\lambda_{\max }=\max _{t>0, \boldsymbol{u}_{0}^{\prime}}\left\|\boldsymbol{u}^{\prime}\right\|_{s} /\left\|\boldsymbol{u}_{0}^{\prime}\right\|_{i}
$$

and the specific $\boldsymbol{u}_{0}^{\prime}$ at which this maximum is attained. If $\|\cdot\|_{i}$ and $\|\cdot\|_{s}$ coincide, then the generalized optimal perturbation problem reduces to the optimal perturbation problem in its usual formulation. 
Since (3.1), (3.3) is linear, the dependence of $\boldsymbol{u}^{\prime}$ on $\boldsymbol{u}_{0}^{\prime}$ can be expressed as $\boldsymbol{u}^{\prime}=A(t) \boldsymbol{u}_{0}^{\prime}$, where $A(t)$ is a linear operator depending on time $t$. Finding the optimal perturbation can be done in two steps, first maximizing $\left\|\boldsymbol{u}^{\prime}(t)\right\|_{s}$ over $\boldsymbol{u}_{0}^{\prime}$ with $t$ fixed and then maximizing the result over $t>0$. Assuming that scalar products $(., .)_{i},(., .)_{s}$ can be defined such that $\left\|\boldsymbol{u}^{\prime}\right\|_{i}^{2}=\left(\boldsymbol{u}^{\prime}, \boldsymbol{u}^{\prime}\right)_{i},\left\|\boldsymbol{u}^{\prime}\right\|_{s}^{2}=\left(\boldsymbol{u}^{\prime}, \boldsymbol{u}^{\prime}\right)_{s}$, maximizing over $\boldsymbol{u}_{0}^{\prime}$ reduces to the variational problem whose functional is

$$
F=\left(A(t) \boldsymbol{u}_{0}^{\prime}, A(t) \boldsymbol{u}_{0}^{\prime}\right)_{s}+\lambda\left(\left(\boldsymbol{u}_{0}^{\prime}, \boldsymbol{u}_{0}^{\prime}\right)_{i}-1\right) .
$$

Here, $\lambda$ is the Lagrange multiplier for the fixed norm of initial conditions.

If, in the numerical implementation, the velocity $\boldsymbol{u}_{0}^{\prime}$ is represented (say, by Galerkin approximation or by a grid function) as a vector $\gamma$ of finite dimension, then we can introduce matrices $E_{t}$ and $E_{0}$ such that $\left(A(t) \boldsymbol{u}_{0}^{\prime}, A(t) \boldsymbol{u}_{0}^{\prime}\right)_{s}=\boldsymbol{\gamma}^{*} E_{t} \boldsymbol{\gamma}$ and $\left(\boldsymbol{u}_{0}^{\prime}, \boldsymbol{u}_{0}^{\prime}\right)_{i}=\boldsymbol{\gamma}^{*} E_{0} \boldsymbol{\gamma}$. Here, ${ }^{*}$ denotes transposition. Then the Euler-Lagrange equation for (3.7) is

$$
E_{t} \boldsymbol{\gamma}+\lambda E_{0} \boldsymbol{\gamma}=0
$$

This is a generalized eigenproblem whose (necessary real) eigenvalues $\lambda$ are the ratios of the square of the norm $\|.\|_{s}$ of the solution at time $t$ to the square of the norm $\|.\|_{i}$ of the initial condition. The notation here is chosen to coincide with notation in equation (22) in Butler \& Farrell (1992) in order to emphasize the similarity. Because of this similarity, many optimal perturbation codes can be modified easily for solving the generalized optimal perturbation problem. The technical and more mathematical details of calculating the generalized optimal perturbation are given in Appendix B.

The selection of the correct solution norm $\|.\|_{s}$ is more important than the selection of the initial condition norm $\|.\|_{i}$. The entire approach of the second framework is based on the assumption that the structure-forming properties of the linear operator dominate. In terms of the illustrative model of $\S 1.3$, this corresponds to the assumption that the peak of $\left|\tilde{\lambda}_{n}\right|$ is significantly narrower than the peak of $\left|\tilde{f}_{n}\right|$ (see figure $3 b$ ). For that model, the generalized optimal perturbation can be formulated as the problem of maximizing $\|\tilde{u}\|=\sum_{n} \tilde{u}_{n}^{2}=\sum_{n} \lambda_{n}^{2} \tilde{w}_{n}^{2}$ over all $\tilde{w}_{n}$ such that $\|\tilde{w}\|_{i}=\sum_{n} \tilde{w}_{n}^{2} / \alpha_{n}^{2}=1$. Here different sets of $\alpha_{n}$ correspond to different initial norms $\|.\|_{i}$. In the limiting case of a very narrow peak of $\left|\tilde{\lambda}_{n}\right|$, the wavelength of the dominant mode of $\tilde{u}(z)$ will coincide with the wavelength of the generalized optimal perturbation for any reasonable set of $\alpha_{n}$. This is why the initial norm is less important.

Note that even when the peak of $\left|\tilde{\lambda}_{n}\right|$ is not narrow, the wavelength of the generalized optimal perturbation will coincide exactly with the dominant wavelength of $\tilde{u}$ if we take $\alpha_{n}=\left|\tilde{f}_{n}\right|$. Of course, if $\tilde{f}_{n}$ were known, there would be no need to solve the optimization problem. It is clear, however, that if the peak of $\left|\tilde{\lambda}_{n}\right|$ is narrow but finite, and there is some limited information on $\tilde{f}_{n}$, this information can be taken into account by the appropriate selection of $\|.\|_{i}$.

In the case of the flow in a plane channel, the optimal perturbation will be characterized not only by the spanwise wavenumber similar to $n$ in the above illustration, but also by the longitudinal wavenumber. It will also be dependent on the wall-normal coordinate. To test the predictive force of the approach, we will compare the spanwise period of the generalized optimal perturbations with the streak spacing observed in physical and numerical experiments. To ensure the purity of the comparison, we will not use any information on any spanwise scales as the input data. Also, since all optimal perturbations for channel flows found so far are independent of longitudinal coordinates, for simplicity we will limit the analysis to such perturbations. This eliminates the longitudinal wavenumber. However, since the 
implied physical mechanism involves mean shear, we have to prescribe the mean velocity profile $U(y)$, and since it also involves the lift-up of the mean profile of the quantity for which the streaks spacing is being predicted (velocity or passive scalar), we have to prescribe the mean profile of that quantity, too. In this situation, it appears to be reasonable to allow an input of other information on the distribution of parameters of a turbulent flow as a function of the wall-normal coordinate, and the initial condition norm is the natural way of incorporating that information. Two initial condition norms were considered.

The simplest initial condition norm is the usual $L_{2}$ norm, that is the cross-flow perturbation energy density averaged over the entire channel

$$
\|\boldsymbol{u}\|_{c}^{2}=\lim _{a, b \rightarrow \infty} \frac{1}{a b} \int_{x, y, z=0,-1,0}^{x, y, z=a,+1, b}\left(v^{2}+w^{2}\right) \mathrm{d} x \mathrm{~d} y \mathrm{~d} z .
$$

We assume that the channel walls are at $y= \pm 1$. Optimal perturbations for channel flow usually have a zero longitudinal velocity component at $t=0$. For simplicity, we assume this to be true for the generalized optimal perturbation. This is the reason why the right-hand side of (3.9) does not involve $u$.

The norm (3.9) remains finite even for $v$ and $w$ not satisfying the no-slip and impermeability conditions at the wall. It would be reasonable, however, to use the initial condition norm in order to eliminate at least such unrealistic $v$ and $w$ from the analysis. Moreover, in a turbulent flow, the mean energy of the cross-flow motion strongly depends on the distance to the wall. The initial norm is the natural tool for taking this into account. This can be done by using the norm

$$
\|\boldsymbol{u}\|_{d}^{2}=\lim _{a, b \rightarrow \infty} \frac{1}{a b} \int_{x, y, z=0,-1,0}^{x, y, z=a,+1, b}\left(\frac{v^{2}}{\left\langle v^{2}\right\rangle}+\frac{w^{2}}{\left\langle w^{2}\right\rangle}\right) \mathrm{d} x \mathrm{~d} y \mathrm{~d} z
$$

Here, $\left\langle v^{2}\right\rangle(y)$ and $\left\langle w^{2}\right\rangle(y)$ are the normal Reynolds stresses, which can be taken from experiment or direct numerical simulation. Intuitively, as compared to $\left\|\left(v_{0}, w_{0}\right)\right\|_{c}=1$, the restriction $\left\|\left(v_{0}, w_{0}\right)\right\|_{d}=1$ allows greater values of $v_{0}$ and $w_{0}$ where $\left\langle v^{2}\right\rangle$ and $\left\langle w^{2}\right\rangle$ are large and requires smaller values of $v_{0}$ and $w_{0}$ where $\left\langle v^{2}\right\rangle$ and $\left\langle w^{2}\right\rangle$ are small. In addition, it has a neat form with the initial optimal perturbation energy weighted with the turbulent energy of the real flow.

More involved argument in favour of (3.10) is given in the Appendix B. That argument also suggests that the linear functional describing the dependence of the solution of the linearized Navier-Stokes equations, written as an $L_{2}$ scalar product of the initial condition and the Green function, should be extended to the initial conditions $\boldsymbol{u}_{0}$ not necessarily satisfying the continuity equation, and that the optimization should be performed over all such initial conditions. This strengthens the point made earlier that only the part of the optimal perturbation that was maximized in amplitude can be expected to be close to the characteristics of the real flow.

The input required for the calculation of the generalized optimal perturbation consists of the Reynolds number $R e$, the coordinate of the visualization plane $y$ (it enters (3.5)), the mean shear profile $U(y)$, and the mean wall-normal gradient of the quantity for which the streaks spacing is being predicted, that is $\mathrm{d} U / \mathrm{d} y$ for the velocity streaks. (For scalar streaks considered later it is replaced with $\mathrm{d} \Theta / \mathrm{d} y$.) If the norm $\|.\|_{d}$ is used, the normal Reynolds stresses $\left\langle v^{2}\right\rangle(y)$ and $\left\langle w^{2}\right\rangle(y)$ are also required. There are no adjustable parameters. 

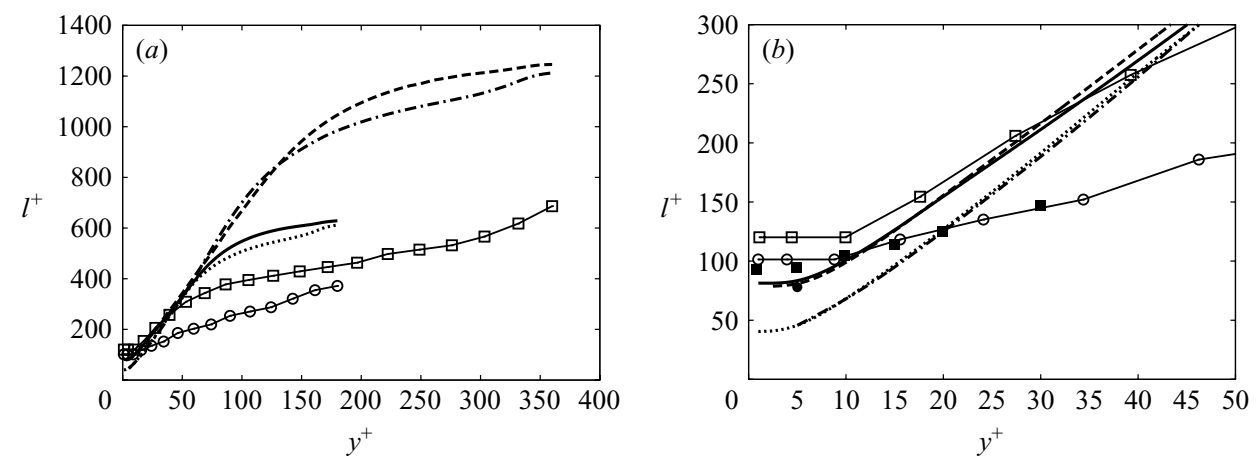

FIGURE 5. Streak spacing as a function of the distance to the wall. -, GOP using $\|\cdot\|_{d, 1 D}, R e=180 ;---360 ; \cdots$, GOP using $\|\cdot\|_{c, 1 D}, R e=180 ;-\cdot-\cdot, 360 ; \bigcirc$, Hu \& Sandham, $R e=180$, from autocorrelation; $\square, 360 ; \boldsymbol{\square}$, Smith \& Metzler, $\operatorname{Re}_{\theta}=2030$, mean; $\bullet$, Smith \& Metzler, most probable.

\section{Comparisons}

\subsection{Velocity streaks}

The mean velocity distribution of the turbulent flow was approximated with the Reynolds-Tiederman profile used also by Butler \& Farrell (1993). The normal Reynolds stresses $\left\langle v^{2}\right\rangle$ and $\left\langle w^{2}\right\rangle$ were taken from the direct numerical simulation results of Hu \& Sandham (2001).

The Mathematica package was used for calculating explicitly the eigensolutions of the linearized Navier-Stokes equations and for prototyping the more efficient Fortran code. The code was validated by reproducing the results of Butler \& Farrell (1993), and then, by small modification, it was adjusted for solving the generalized problem. More detail is given in the Appendix C.

Predictions of the generalized optimal perturbation approach were compared with the results of direct numerical simulation of a channel flow by Hu \& Sandham (2001; see also Hu, Morfey \& Sandham 2003) for two values of the Reynolds number based on the skin friction and the channel half-width, and with the experiment of Smith \& Metzler (1983). The experimental measurements were performed in a boundary layer, with the Reynolds number $R e_{\theta}$ based on the momentum thickness and the upstream velocity. If the dynamic velocity were used, their Reynolds number would be roughly about 100, and if, in addition, the boundary-layer thickness were used instead of the momentum thickness, then it would be roughly about 1000 .

In direct numerical simulation and experiments, streaks are not exactly regular, and different definitions of streak spacing are possible. The velocity autocorrelation function $R_{u u}(\Delta)=\langle u(t, x, y, z) u(t, x, y, z+\Delta)\rangle$ has a maximum at $\Delta=0$ and reaches a minimum at a certain value $\Delta_{\min }$. Streak spacing can be defined as $2 \Delta_{\min }$. Note that both $R_{u u}(\Delta)$ and $\Delta_{\min }$ depend on $y$, but are independent of $t, x$, and $z$. This definition was accepted for the direct numerical simulation results. Smith \& Metzler (1983) visually identified streaks in photographs obtained in the experiment and determined the probability density distribution for the streak spacing. The mean and the most probable streak spacing were then calculated from this distribution. The generalized optimal perturbations are periodic in the spanwise direction, and the streak spacing equals the period.

Figure 5(a) shows the comparison of the generalized optimal perturbation results with direct numerical simulations for the entire channel width and figure $5(b)$ shows 
also the experimental data, but only for the near-wall region. Streaks observed in experiments and direct numerical simulations are a near-wall feature. Away from the walls, the minimum of the autocorrelation function is affected by the flow structures with a relatively short longitudinal scale. In contrast, in the present study, the generalized optimal perturbation is limited to 'streaky' perturbations independent of $x$. Therefore, away from the wall they cannot be compared. In principle, however, it is possible to filter the structures with large longitudinal scales in direct numerical simulation and then compare their spanwise scale with the generalized optimal perturbation predictions. In part, figure 5(a) is given for the purposes of such future comparisons. This figure also shows that the generalized optimal perturbations obtained with two different initial condition norms give similar results.

Figure $5(b)$ gives more detailed comparison in the near-wall region where performing the quantitative comparison makes sense. As can be expected, the generalized optimal perturbation with $\|.\|_{d}$ gives a better agreement, and as also can be expected, the generalized optimal perturbation agrees better with the most probable value of the streak spacing. Qualitatively, the generalized optimal perturbation results and the numerical results differ in that, even for $y^{+}<50$, the generalized optimal perturbation results for the two values of the Reynolds number almost overlap while the numerical results depend on $R e$. However, as far as dependence on the wall distance is concerned, the generalized optimal perturbation reproduces both the flat portion of the $l^{+}\left(y^{+}\right)$curve in the near-wall vicinity and the linear increase in $l^{+}$with $y^{+}$further away from the wall. Overall, the comparison seems to be favourable. Both versions of generalized optimal perturbation approach give better results than any other theory. Indeed, to the best of our knowledge, none of the existing theories can predict the variation of streak spacing with the distance to the wall. For comparison, the optimal perturbation without the eddy-turnover-time adjustment gives $l^{+}=540$ independent of $y^{+}$for $R e=180$ (Butler \& Farrell 1993). Even with the eddy-turnovertime adjustment the optimal perturbations of Butler \& Farrell give the same streak spacing in all visualization planes.

\subsection{Selectivity of GOP}

By its very nature, the generalized optimal perturbation approach is only approximate, as figure $3(b)$ illustrates by the difference in the position of the maximums of $\left|\tilde{u}_{n}\right|$ and $\left|\tilde{\lambda}_{n}\right|$. In order to judge better the strength of the evidence given by the comparison in figure $5(b)$, it would be beneficial to compare the contribution to streaks from the patterns of wall normal motions and from the pattern-forming properties of the linearized Navier-Stokes operator, similarly to the way it was illustrated by figure 3 for the simple model of $\S 1.3$. The case of the real turbulent flow is, however, more complicated. First, in the real case, the parameters additionally depend on the distance to the wall and on the longitudinal coordinate. The generalized optimal perturbation depends also on time. Secondly, there is no information on the behaviour of $\boldsymbol{F}$ in (3.2) from direct numerical simulation or physical experiment. Therefore, it is not possible to plot a curve similar to $\left|\tilde{f}_{n}\right|$ in figure 3 . We can, however, consider the selectivity of the generalized optimal perturbation on its own. If in (3.6) the maximization is performed only over the initial conditions of a specific spanwise period $l^{+}$, then the result will depend on the period; $\lambda=\lambda\left(l^{+}\right)$, and then $\lambda_{\max }=\max \lambda\left(l^{+}\right)$. Figure 6 shows the curves of $\lambda\left(l^{+}\right) / \lambda_{\max }$ for two distances from the visualization plane to the wall, obtained with $\|.\|_{d}$. Note that the shape of these curves is similar to the shape of the probability density function for the streak spacing obtained by Smith \& Metzler (1983). The maxima of $\lambda\left(l^{+}\right)$correspond to the generalized optimal perturbation 


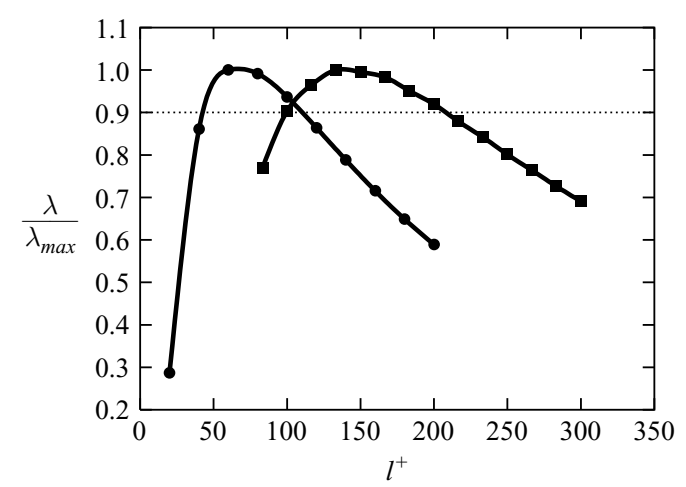

FIGURE 6. Selectivity of the linearized Navier-Stokes operator. $\mathbf{a}, y^{+}=17.62 ; \bullet, 4.43$.

predictions for the streak spacing in figure 5. However, if a $10 \%$ deviation of $\lambda$ from $\lambda_{\max }$ is allowed, then the corresponding variation in $l^{+}$is as large as $50 \%$.

The variation of the streak-spacing values obtained with the use of different definitions, in direct numerical simulations or physical experiment, only increases this uncertainty. For example, the most probable and the average streak spacing measured in the same experiment by Smith \& Metzler (1983) differ by about $20 \%$. For these reasons, we may allow at least $30 \%$ discrepancy between the generalized optimal perturbation predictions and numerical or experimental results. However favourable the qualitative comparison of figure 5 may be, because of this margin, the basis of the quantitative comparison must be extended considerably before reliable conclusions can be made. Passive-scalar streaks provide the material for such extended comparisons.

\subsection{Passive-scalar results}

For perturbations independent of the longitudinal coordinate $x$, the equation for the longitudinal component $u^{\prime}$ of the perturbation velocity has the form

$$
\frac{\partial u^{\prime}}{\partial t}+U \frac{\partial u^{\prime}}{\partial x}+v^{\prime} \frac{\mathrm{d} U}{\mathrm{~d} y}=\frac{1}{R e} \nabla^{2} u^{\prime}
$$

and the equation for the passive scalar perturbation $\theta^{\prime}=\theta-\Theta(y)$ is

$$
\frac{\partial \theta^{\prime}}{\partial t}+U \frac{\partial \theta^{\prime}}{\partial x}+v^{\prime} \frac{\mathrm{d} \Theta}{\mathrm{d} y}=\frac{1}{R e} \nabla^{2} \theta^{\prime}
$$

where $\Theta(y)$ is the mean scalar profile. In our direct numerical simulations, the boundary conditions for the scalars were the same as the boundary condition for the velocity, $\theta^{\prime}=0$ at the walls. To predict the scalar streak spacing using generalized optimal perturbation, we must maximize the norm of $\theta^{\prime}$ rather than the norm of $u^{\prime}$. Since (4.1) and (4.2) differ only in the term with $\mathrm{d} \Theta / \mathrm{d} y$, the same generalized optimal perturbation code can be used for predicting the scalar streaks or the velocity streaks, with $\mathrm{d} \Theta / \mathrm{d} y$ or $\mathrm{d} U / \mathrm{d} y$, respectively, supplied as the input. The generalized optimal perturbation predictions for passive scalars were obtained with the initial condition norm $\|.\|_{d}$.

To ensure as extensive basis for comparisons as possible, the direct numerical calculation of the flow in a plane channel was performed with several passive scalars simultaneously present in the flow and having different mean profiles $\Theta(y)$. Figure 7 summarizes the results for seven scalars. The exact formulae for the shape of each 

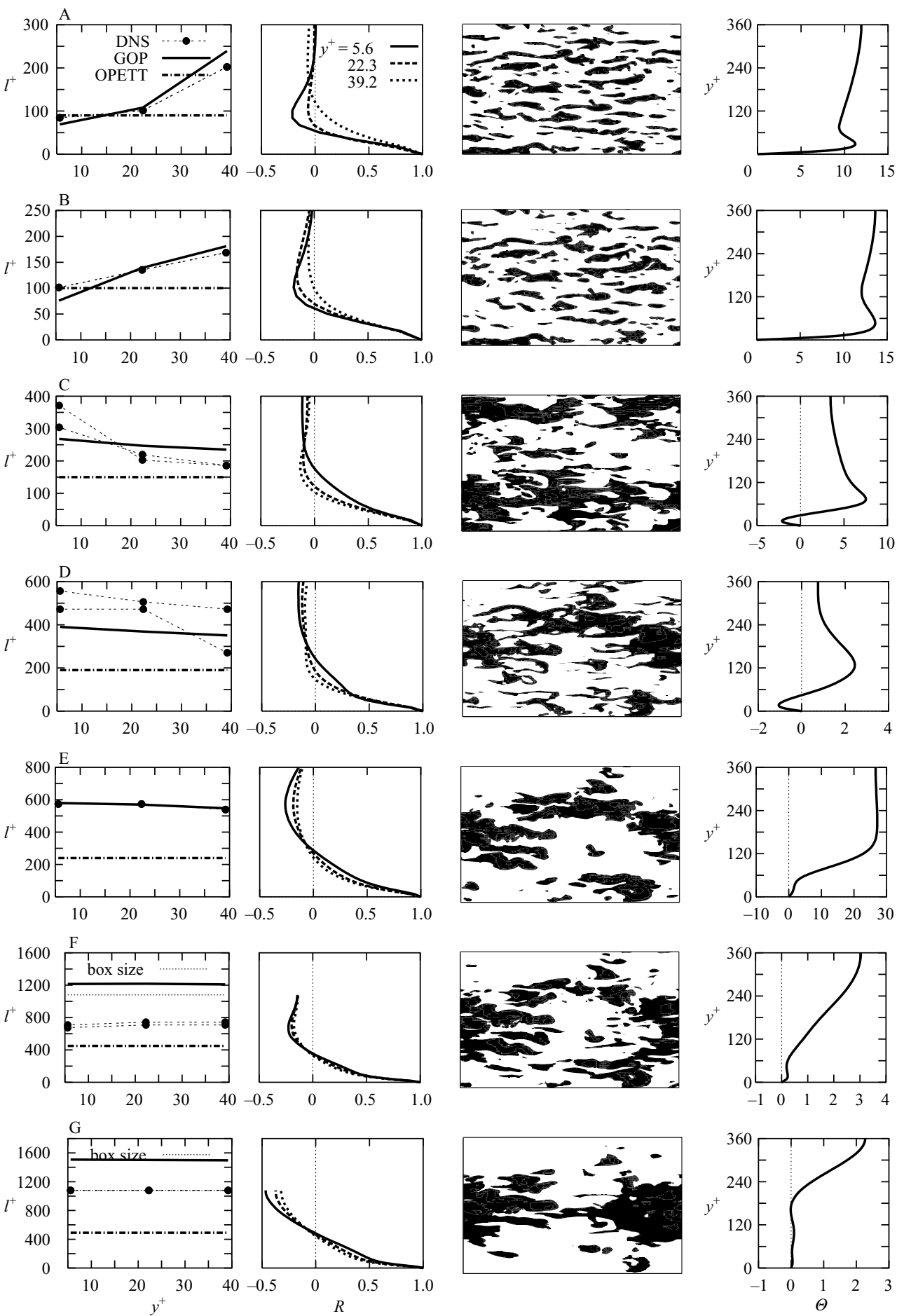

FIGURE 7. Instantaneous visualizations and relations between the streak spacing $l^{+}$, wall distance $y^{+}$, autocorrelation $R$, and mean scalar $\Theta$ for profiles A-G. 
profile and the method of selection of these profiles for comparison are given in Appendix A.

The calculations were performed in a box of size $6 \times 2 \times 3$, in other words, the solutions are double-periodic with period 6 in the main flow direction and with period 3 in the transverse direction, with distance between the walls equal to 2 . The Reynolds number was $R e=360$. The mean pressure gradient was -1 , and, correspondingly, the mean wall shear stress was equal to unity. Accordingly, one wall unit was equal to $1 / 360$. Wall units are used in figure 7 since streak spacing is normally expressed in wall units.

Each row corresponds to the mean scalar profile shown in the last column, with the mean scalar $\Theta$ plotted along the abscissa. The first column shows the streak spacing $l^{+}$as the function of the distance $y^{+}$to the wall, as obtained from direct numerical simulation (DNS), from generalized optimal perturbation theory with $\|.\|_{d}(\mathrm{GOP})$ and from the optimal perturbation theory with eddy turnover time (OPETT) of Butler \& Farrell. In the direct numerical simulation the streak spacing was defined as twice the distance to the minimum of the autocorrelation function. The autocorrelation function $R_{\theta \theta}(\Delta)$ is plotted along the abscissa in the second column of figure 7, for three values of the wall distance. The scale of the ordinate is such that the smallest value of $R_{\theta \theta}$ on the curve is level with the corresponding DNS point on the left of it. For example, in the first row, this level is just below 100 for the solid curve with $y^{+}=5.6$. The third column of figure 7 shows instantaneous visualization of streaks. The flow is from left to right, and the picture plane is parallel to the channel walls. The black areas correspond to regions in the $y^{+}=5.6$ plane where the instantaneous values of $\theta$ were below the threshold values which were adjusted so that visually the area of the dark region was approximately the same in all pictures. For profiles $A-G$ the thresholds values were respectively $15,16,4.5,5,3.6,0.7$ and $1.5 \%$ of the scalar minimal instantaneous value in that plane. All visualization pictures correspond to the same time instant and visualization plane.

For profiles $\mathrm{A}$ and $\mathrm{B}$, the agreement between direct numerical simulation results and the predictions of the generalized optimal perturbation theory is quite good. Note that while, for both profiles, the streak spacing $l^{+}$increases with the distance $y^{+}$from the wall, the average $\mathrm{d}^{2} l^{+} / \mathrm{d} y^{+2}$ is greater for profile A than for profile B. Generalized optimal perturbation results exhibit the same behaviour. In contrast, the optimal perturbation theory with eddy turnover time predicts the same streak spacing for all $y^{+}$. The visualization shows streaky structures in both cases A and B. It should be noted that as the distance to the wall increases, streaks become less apparent (less elongated). The visualizations for greater $y^{+}$are not shown here since this behaviour is well known for velocity streaks (Smith \& Metzler 1983).

In contrast to $\mathrm{A}$ and $\mathrm{B}$, for profiles $\mathrm{C}$ and $\mathrm{D}$ the streak spacing decreases as $y^{+}$ increases. This behaviour also is reproduced by generalized optimal perturbation, but the quantitative agreement is less good. Note the shape of the autocorrelation curves; in these cases it is difficult to determine the location of the minimum of the autocorrelation function. The direct numerical simulation data shown were obtained by time-averaging. The averaging time $T_{a v}$ was repeatedly increased until the variation of autocorrelation function with $T_{a v}$ fell to within about $3 \%$ in the worst case. However, where the lowest part of the autocorrelation curve is almost flat, even a small variation in $R_{\theta \theta}(\Delta)$ can result in a large variation of the location of the minimum. To illustrate the uncertainty caused by this effect, each plot in the first column shows, in fact, the direct numerical simulation results for two values of $T_{a v}$, but in cases A, B, E and G they overlap, and in case F they are quite close. In cases 


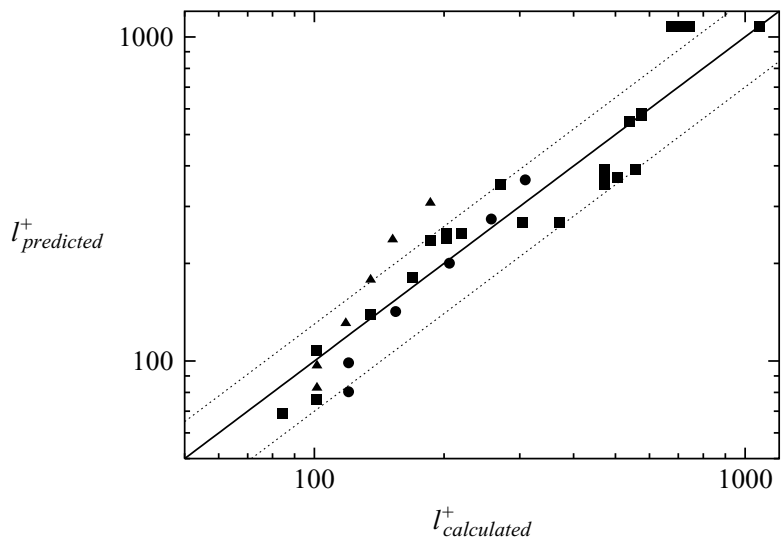

FIGURE 8. Overall comparison of predicted vs. calculated streak spacing, $y^{+}<50$. $\mathbf{m}$, scalars, $R e=360 ; \boldsymbol{\Lambda}$, velocity, $R e=180 ; \bullet$, velocity, $R e=360 ;-, D N S ; \cdots, 30 \%$ margin.

$\mathrm{C}$ and $\mathrm{D}$, however, the uncertainty in direct numerical simulation results is of the same order of magnitude as the deviation of these results from generalized optimal perturbation predictions. In case E, the minimum of the autocorrelation function is clear, and the agreement between the direct numerical simulation and generalized optimal perturbation is again quite good.

In cases $\mathrm{F}$ and $\mathrm{D}$, the agreement is affected by the finite size of the computational box. The maximum streak spacing that could be obtained in our direct numerical simulation is equal to the box size of 1080 wall units. In both cases, the generalized optimal perturbation approach predicts streak spacing greater than this value, and, of course, if the possible perturbations were limited to wavelengths not greater than the box size, then generalized optimal perturbation would give just the box size as the optimal perturbation. Nevertheless, in case F, the direct numerical simulation result is below 1080. It should be noted, of course, that an insufficient box size affects the results not only by limiting the maximum possible wavelength, but also by distorting the spectrum in the vicinity of the box size. This can explain the discrepancy. There is also another possibility. The streak visualizations clearly show that as the streak spacing increases from profile A to profile $G$, the streaks become less and less discernible. On the other hand, the generalized optimal perturbations considered here are limited to perturbations independent of $x$, that is, elongated streaks. Therefore, there is a possibility that removing the limitation of $\partial / \partial x=0$ for generalized optimal perturbations may further improve the agreement.

Overall quality of generalized optimal perturbation predictions can be judged by figure 8 . The abscissa of each plotted point is the streak spacing obtained from direct numerical simulation for the same scalar profile and the ordinate is the value predicted by the generalized optimal perturbation approach for the same case. Results for velocity streaks are also shown. When the predicted and the calculated values coincide, the point is on the diagonal shown with a solid line. Dotted lines indicate the $\pm 30 \%$ margin. For the three cases when generalized optimal perturbation predicts spacing greater than the size of the computational domain, this prediction was reduced to this size $\left(y^{+}=1080\right)$ when plotting figure 8 .

An important point to be noted from figure 8 is the large variation of streak spacing in direct numerical simulation results. Even limited to profiles A, B and C (the profiles resulting in clearly manifested streaks) the variation (from 85 to more than 300 ) of the 
streak spacing is well beyond what could be considered as compatible with the idea that these different streaks are dictated by the same pattern of wall-normal motions.

Part of the observed deviation of the predicted results from the numerical results should be attributed to the approximate nature of the calculations we performed within the generalized optimal perturbation approach. However, even if this deviation is attributed exclusively to the influence of the pattern of the wall-normal motions not accounted for by the generalized optimal perturbation approach, it nevertheless is only about $30 \%$. Therefore, for at least the remaining $70 \%$, the streak spacing is dictated by the structure-forming properties of the combined action of the lift-up of the mean profile, mean shear and diffusion.

\section{Discussion and conclusions}

\subsection{The physical mechanism of the spanwise scale selection}

The physical mechanism of the selection of a certain streak spacing of an optimal perturbation can be described in the following way. An optimal perturbation consists of longitudinal vortices of a fixed initial energy and zero initial longitudinal velocity. The longitudinal vortices decay with time, while longitudinal velocity perturbation grow to quite large amplitude before starting to decay. This growth is caused by the wall-normal velocity component of the optimal vortex, which lifts up the slowly moving fluid from the wall and advects the fast-moving fluids to the wall in alternating stripes. For perturbations of small spanwise wavelength, viscosity causes faster decay of the longitudinal vortices and smoothes out the non-uniformity in longitudinal velocity. Because of this, for very small wavelengths, the streak amplitude is small. For very large spanwise wavelengths, almost all kinetic energy of the longitudinal vortex is concentrated in the spanwise velocity component, while the lift-up effect is proportional to the wall-normal velocity component. Because of this, for very large wavelengths, the streak amplitude is also small and, hence, there is a maximum in between.

In a form directly applicable to turbulent flow and not only to perturbations governed by linearized Navier-Stokes equations used in the generalized optimal perturbation theory, the streak scale selection mechanism can be formulated in the following way. Streaks appear owing to wall-normal motions advecting the slowly moving fluid away from the wall. The streak spacing is determined by the balance between viscous diffusion, which strengthens as the streak spacing decreases, and vanishing of the wall-normal component of velocity fluctuations as the streak spacing increases.

\subsection{Generalized optimal perturbation for longitudinal vortices}

The generalized optimal perturbation and the developed turbulent flow can be expected to have in common only that specific feature the amplitude of which was maximized when generalized optimal perturbation was determined. The generalized optimal perturbation of $\S 3.3$, that is calculated by maximizing the norm of the longitudinal velocity $\|u\|_{y}$, has a structure similar to figure 1(b), that is, apart from streaks it also involves longitudinal vortices. However, the magnitude of these vortices is not large and, unlike streaks, their presence in this generalized optimal perturbation gives no reason to expect them to be observed in the real flow.

By the very idea of generalized optimal perturbation, in order to determine what kind of longitudinal vortices will be observed in the real flow, the solution norm should be tailored so as to measure the amplitude of longitudinal vortices rather than 
streaks. The simplest such norm is just the kinetic energy of the cross-flow motion, with the additional assumption of the flow being independent of longitudinal coordinate required in order to emphasize the longitudinal vortices. However, an attempt to find the corresponding generalized optimal perturbation immediately reveals the wellknown fact that longitudinal vortices have no transient growth. The reason for this is that all $x$-independent eigensolutions of the linearized problem are orthogonal with respect to the cross-flow energy norm. Technically, we can find the longitudinal-vortextailored generalized optimal perturbation, but it is just the slowest-decaying vortex. Of course, the longitudinal vortices of the generalized optimal perturbation based on the streak-tailored solution norm $\|.\|_{y}$ decay even faster. Therefore, within the linearized Navier-Stokes equations framework, the preferential longitudinal vortex spacing is the spacing between the slowest-decaying vortices. Such vortices have a period of approximately $2 \pi / 1.2$ times the channel half-width independently of the Reynolds number (see, for example, Waleffe \& Kim 1997), which is much larger than the characteristic spanwise scale in the near-wall region of a real flow.

Naturally, since these vortices are only decaying, the nonlinear effects may well prevail in real flow as far as vortices are concerned. In general, generalized optimal perturbation determines the structure most favoured by the linearized Navier-Stokes equations. Whether this structure will dominate also the solution of the full nonlinear Navier-Stokes equations may depend on its strength characterized by the absolute value of $\lambda_{\max }$. There is a certain difficulty in interpreting specific values of $\lambda_{\max }$ in the case of generalized optimal perturbation owing to the difference between the initial norm and solution norm. A correlation between $\lambda_{\max }$ and the degree to which streaks were pronounced in figure 7 was indeed observed, but further work in this direction is desirable.

If the restriction $\partial / \partial x=0$ is not imposed, we can expect the possibility of transient growth even with respect to the norm tailored to vortices. It would be interesting to calculate the vortical structures using a generalized optimal perturbation approach with the norm tailored to non-longitudinal, that is general, vortices, and compare the result with experimental and numerical observations. In doing this, the solution norm should be chosen with care in order that strong streaks would not obscure other possible structures. Anyway, whatever vortical structures can be revealed by such other generalized optimal perturbation solutions, they need not be directly related to streaks.

\subsection{A note on the origin of structure in turbulent flows}

There are two possible concepts of the origin of structure in developed turbulent flows. One obvious idea implies the existence of a closed cycle of regeneration of structures, in which, say, hairpin vortices produce streaks and streaks in turn produce hairpin vortices and so on. The cycle may include more types of structure than these two. In this concept, none of the structures is primary. Another possibility is that certain primary structures appear directly from an unstructured background. They can produce other structures and so on until the last structure in the chain is dissipated by viscosity or destroyed by the chaotic motion. The results of the present paper favour this second possibility and indicate that streaks may be such a primary structure. Naturally, more complicated concepts, say, with several independent cycles and/or chains are also possible. Note that while the chain of the structure regeneration may not be closed, the turbulence kinetic energy regeneration cycle remains closed and the closing link is formed by motions having no dominant pattern. 
The vortices produced by streak instabilities are likely to inherit from streaks the same spacing. Such vortex production by streaks is likely to require nonlinearity to play a role. As we can see from the generalized optimal perturbation approach applied to longitudinal vortices, linear effects alone favour longitudinal vortices of much greater spacing (see § 5.2). Therefore, if the nonlinearity plays a key role in the generation of vortices from streaks, then switching off the nonlinear terms should lead to an increase in vortex spacing, as indeed was observed by Kim \& Lim (2000), but should not affect strongly the streak spacing until much later times when the solution will approach steady flow. However, Kim \& Lim (2000) do not give data on longitudinal velocity.

The presence of a variety of structures, including the so-called organized vortices, in near-wall turbulent flows appears to be a well-established fact. It is more difficult to prove definitely by numerical or experimental observations the existence of a relationship between organized vortices and streaks. An observation that characteristic spacing between organized vortices is equal to the streak spacing could provide such a proof. However, we have already mentioned that, for example, the wall-normal velocity has a characteristic spanwise wavelength of only about half of the streak spacing. Of course, even if streaks are not generated by organized vortices, the process of vortex generation by streaks could explain the correlation between them. At the same time, identification of such vortices by an observer is subjective. Being influenced by the widely accepted view that organized vortices are necessary for the generation of streaks (figure $1 b$ ) and because of the solid evidence of the existence of streaks (figure 1a) an observer can wishfully pick up vortical structures where in fact there are none in a chaotic picture of the distribution of, say, vorticity or pressure, or of any other criteria used for the purpose. In view of the results of the present study, it seems reasonable to re-examine cautiously the available evidence of the existence of correlation between vortices and streaks, with more emphasis on objective techniques. It might well be instructive to apply the techniques used for discovering vortical patterns to an unstructured reference velocity field similar to the mean flow plus random potential perturbations of $\S 2.1$, maybe with added coherent streaks.

\subsection{A unifying hypothesis for the second conceptual framework}

Works using linearized Navier-Stokes equations for describing features of turbulent flows are sometimes based on assumptions that are not really valid for turbulent flows, or simply do not give any justification for the use of the linearized equations, apart from the comparison with experiment or direct numerical simulations. We can now put a number of such studies into the context of the second conceptual framework. Alongside the full Navier-Stokes equations, (3.1)-(3.3), written in perturbation variables $\boldsymbol{u}^{\prime}=\boldsymbol{u}-\boldsymbol{U}$ and $p^{\prime}=p-P$, we also consider the linearized Navier-Stokes equations with a forcing term $\sigma=\sigma(t, x)$ :

$$
\left.\begin{array}{c}
\frac{\partial \boldsymbol{v}^{\prime}}{\partial t}+\boldsymbol{U} \cdot \nabla \boldsymbol{v}^{\prime}+\boldsymbol{v}^{\prime} \cdot \nabla \boldsymbol{U}+\nabla q^{\prime}-\frac{1}{R e} \nabla^{2} \boldsymbol{v}^{\prime}=\sigma, \\
\nabla \boldsymbol{v}^{\prime}=0 .
\end{array}\right\}
$$

Note that the solution $\boldsymbol{u}^{\prime}$ of (3.1)-(3.3) is equal to the solution of (5.1) with $\boldsymbol{\sigma}=\boldsymbol{F}$ (see (3.1)). We then introduce an object of interest $I=I\left[\boldsymbol{u}^{\prime}\right]$ which depends on $\boldsymbol{u}^{\prime}$. In general, $I$ can be of arbitrary nature, but in the simplest case, it can be a quantity, for example, the average streak spacing in a given visualization plane. We assume that there is a suitable space with a norm making it possible to compare $I\left[\boldsymbol{u}^{\prime}\right]$ and $I\left[\boldsymbol{v}^{\prime}\right]$ by 
calculating $\left\|I\left[\boldsymbol{u}^{\prime}\right]-I\left[\boldsymbol{v}^{\prime}\right]\right\|$. Let $S$ be a set of functions $\sigma$ such that $\left\|I\left[\boldsymbol{v}^{\prime}\right]-I\left[\boldsymbol{u}^{\prime}\right]\right\|<\varepsilon$ for any $\sigma \in S$, where $\boldsymbol{v}^{\prime}$ is the solution of (5.1).

We suggest the following hypothesis underlying the second conceptual framework: for many objects of interest $I$ (and, in particular, for $I$ equal to the streak spacing) the set $S$ is quite large in a certain sense even for relatively small $\varepsilon$. If this is indeed the case, then for obtaining $I\left[\boldsymbol{u}^{\prime}\right]$ with error less than $\varepsilon$, we do not need to know $\boldsymbol{F}$. It is sufficient to take any $\sigma \in S$, solve (5.1) and calculate $I\left[\boldsymbol{v}^{\prime}\right]$.

Selecting $\sigma$ from physical considerations and solving (5.1) is the procedure performed within the rapid distortion theory (RTD) even though its justification usually given in RDT is different, see, for example Nazarenko, Kevlahan \& Dubrulle (1999), where also the WKB approach was used for solving (5.1) approximately. In other RDT works, $\boldsymbol{\sigma}$ is often taken to be equal to $\delta(t) \boldsymbol{v}_{0}^{\prime}(x)$ so that the forced response problem is reduced to an initial-value problem. Landahl (1989) also selected $\sigma$ from physical considerations, but solved the linearized boundary-layer equations. In other studies, an attempt is made to ensure that $\sigma \in S$ by determining $\sigma$ giving the strongest response. The present study is in this category, and also the studies of Butler \& Farrell (1993) and Carpenter et al. (2003).

Note that, to the best of our knowledge, the hypothesis that for various quantities of interest the set $S$ is indeed large was never put forward explicitly. However, this hypothesis can serve as an alternative unified justification for the number of previous studies. It would be interesting to verify this hypothesis by direct rigorous investigation of the properties of the linearized Navier-Stokes equations.

\subsection{Conclusions}

The present paper considered the two conceptual frameworks for the origin of the streaky pattern in near-wall developed turbulent flows. According to the first framework, the pattern of streaks is dictated by the pattern of wall-normal motions (longitudinal vortices, hairpin vortices, vortex packets, etc.) via the lift-up mechanism. According to the second framework, the pattern of streaks is dictated by the structureforming properties of the combined action of lift-up of the mean profile, mean shear and viscous diffusion, which can be described by the linearized Navier-Stokes equations.

Since the generation of streaks can be logically explained within both conceptual frameworks, the conclusion must be based on other evidence. The objective evidence in favour of the first conceptual framework consists mostly of the experimental observations that vortices are concentrated in the vicinity of streaks. Even if we accept the objectivity of such observations, they can equally be explained by assuming that vortices create streaks or by assuming that streaks create vortices. Therefore, these observations cannot be considered as a valid argument in favour of any of these explanations.

In contrast, there is a considerable amount of evidence in favour of the second conceptual framework. It consists of the observations that linearized Navier-Stokes equations can predict many of the features of the developed turbulent flows, in particular the observations made within the RDT theory and also other works cited in $\S 5.4$, and of the results of the present study. The latter include the numerical experiments showing that streaks can be created by unstructured wall-normal motions (figure 4), the observations of very different streaks of different scalars in the same flow (figure 7), and the qualitative (figures 5 and 7 (left-hand column)) and quantitative (figure 8) comparisons of the generalized optimal perturbation approach results with numerical and physical experiment. 
Therefore, the following conclusions can be made.

(i) Streaks appear mostly because of the structure-forming properties of the combination of lift-up of the mean profile, mean shear and viscous diffusion.

(ii) The pattern or absence of pattern of wall-normal motion is of less significance for streaks.

(iii) A theoretical approach (GOP) based on this idea has a significant predictive capability.

The way of presenting the findings of this study and even their interpretation evolved considerably as a result of the discussions with our colleagues and many of the seminar speakers in the AFM research group University of Southampton, with a number of other workers who kindly provided their feedback over e-mail, and of the referee reports on this and related papers. The authors are very grateful to these people, far too numerous to be named here.

This work was supported in part by the EPSRC grant GR/R27785 on 'Mechanism of longitudinal vortices in near-wall turbulent flow'. M. F. B. acknowledges support via studentship from the School of Engineering Sciences of the University of Southampton. The authors would like to thank Professor N. D. Sandham for providing the code for direct numerical simulations and Dr Z.W. Hu for providing the direct numerical simulation data for the plots in electronic form.

\section{Appendix A. The mean profiles of the passive scalars}

Since the equations for the scalar are linear, a linear combination of several solutions is a solution. If

$$
\theta=\sum_{i=1}^{i=n} A_{i} \theta_{i},
$$

then

$$
R_{\theta \theta}(\Delta)=\sum_{i=1, j=1}^{i=n, j=n} A_{i} A_{j} R_{\theta_{i} \theta_{j}}(\Delta) .
$$

Note that the mean profile of the scalar is

$$
\Theta=\langle\theta\rangle=\sum_{i=1}^{i=n} A_{i}\left\langle\theta_{i}\right\rangle=\sum_{i=1}^{i=n} A_{i} \Theta_{i} .
$$

Therefore, by solving simultaneously several passive-scalar equations with different source terms and calculating the cross-correlations $R_{\theta_{i} \theta_{j}}(\Delta)$, it is possible to calculate the autocorrelation function and, hence, the streak spacing, for any linear combination determined by the vector of coefficients $A_{i}$. To check this concept, one of the calculated scalars was a linear combination of the rest, so that its autocorrelation function was calculated both directly and by the method described above, and the results coincided. We also compared the streak spacing as calculated from the autocorrelation function with the streak spacing as, although rather approximately, obtained from visualization, and again found that our linear-combination approach works.

Technically, it was easier to modify the code in so that instead of prescribing the source terms $S_{i}$ we could prescribe directly the mean scalar profiles $\Theta_{i}(y)=\left\langle\theta_{i}\right\rangle$. Solutions for seven scalar mean profiles were calculated simultaneously, with one, as mentioned, being a linear combination of others for validation purposes. Therefore, 
the results were obtained for six independent basic profiles. For completeness, the best choice of the basic profiles would be a sufficiently large number of, say, Chebyshev polynomials, so that practically any mean profile could be represented as a linear combination of them. However, the required computer resources would be too large. We compromised by taking three profiles of the shape more or less close to what seemed to be of possible interest and three other profiles as trigonometric functions, to allow for further adjustments. The profiles were defined via their derivatives with respect to $y$, because these derivatives are required for optimal perturbation theory. Introducing two auxiliary functions,

$$
g(y, R e)=\frac{1}{2}\left[1+\left(\frac{0.525 \operatorname{Re}\left(1+y^{2}-2 y^{4}\right)(1-\exp (-(1-|y|) \operatorname{Re} / 37)}{3}\right)^{2}\right]^{1 / 2}-0.5,
$$

( $g(y, R e)$ corresponds to the Reynolds-Tiederman profile, as in Waleffe et al. 1993), and

$$
h(y, d, w)=150\left(\exp \left(-(-1+d-y)^{2} / w^{2}\right)-\exp \left(-(-1+d+y)^{2} / w^{2}\right)\right),
$$

the derivatives of the basic mean profiles are

$$
\begin{aligned}
\Theta_{1}^{\prime} & =-y \times \operatorname{Re} /(1+g(y, \operatorname{Re})), \\
\Theta_{2}^{\prime} & =h(y, 0.20,0.150), \\
\Theta_{3}^{\prime} & =h(y, 0.10,0.075), \\
\Theta_{4}^{\prime} & =\sin (1 \times \pi(1+y) / 2), \\
\Theta_{5}^{\prime} & =\sin (3 \times \pi(1+y) / 2), \\
\Theta_{6}^{\prime} & =\sin (5 \times \pi(1+y) / 2) .
\end{aligned}
$$

Cross-correlations were calculated for solutions corresponding to these six profiles. Results of the calculations showed the existence of scalar streaks and moderate variation in their spacing.

For checking to what extent the streak spacing can be varied by varying the mean scalar profile, the following method was used. Ideally, we can try to find such a vector $\left(A_{1}, \ldots, A_{6}\right)$ that the autocorrelation function (A 2) has a minimum at, or as close as possible to, a desired value of $\Delta=\Delta_{0}$. Then, varying this value would vary the streak spacing. Such a problem, however, is too difficult. Instead, we first normalize the autocorrelation function (A 2) of the linear combination (A 1) by imposing a restriction that it is equal to unity at $\Delta=0$ :

$$
R_{\theta \theta}(0)=\sum_{i=1, j=1}^{i=6, j=6} A_{i} A_{j} R_{\theta_{i} \theta_{j}}(0)=1 .
$$

Then we require this normalized autocorrelation function to be as small as possible at $\Delta=\Delta_{0}$. We expect that this function will then have a minimum not too far away from that value, so that the streak spacing can be controlled by $\Delta_{0}$. This idea, while working in many cases, for certain values of $\Delta_{0}$ resulted in an ill-conditioned problem for the following reason. Imagine that the basic profiles are not linearly independent. If $\sum_{i=1}^{i=6} A_{i}^{0} \Theta_{i}=0$, then adding vector $\mathbf{A}^{0}$ multiplied by an arbitrary constant $C$ to the solution $\mathbf{A}$ of our minimization problem will change nothing. Therefore, the solution is not unique and the problem is ill-posed. Notice that $C$ may be very large so that the sum $C \mathbf{A}^{0}+\mathbf{A}$ may be large, too. In practice, in our case, the basic profiles are not linearly dependent, but, on one hand they are not orthogonal, and on the other 


\begin{tabular}{ccccccccc}
\hline & & & & & & & & \\
ID & $2 \Delta_{0}$ & $r$ & $A_{1}$ & $A_{2}$ & $A_{3}$ & $A_{4}$ & \multicolumn{1}{c}{$A_{5}$} & \multicolumn{1}{c}{$A_{6}$} \\
A & 16.87 & 0.1 & -0.9452 & 0.03027 & 0.3191 & 0.003794 & -0.03407 & -0.05132 \\
B & 101.2 & 0.1 & -0.9841 & 0.1426 & 0.04708 & -0.0007388 & -0.08993 & -0.03150 \\
C & 270.0 & 0.01 & -0.3198 & -0.1153 & 0.7649 & -0.1601 & 0.5173 & 0.07813 \\
D & 320.6 & 0.001 & 0.1415 & -0.07865 & -0.07077 & 0.3237 & -0.8607 & -0.3522 \\
E & 438.8 & 0.1 & -0.07543 & -0.8645 & 0.4305 & 0.02091 & -0.1787 & 0.1709 \\
F & 776.2 & 0.0025 & -0.03643 & -0.03295 & 0.002782 & -0.2214 & 0.9641 & 0.1380 \\
G & 1063 & 0.001 & -0.006337 & -0.02004 & 0.005644 & -0.1841 & 0.9090 & -0.3733
\end{tabular}

TABLE A 1. Values of parameters and obtained eigenvectors.

hand, random numerical errors are present. Together, it sometimes leads to very large vectors A determined mostly by the numerical errors, with erratic behaviour of the resulting autocorrelation function. The obvious solution to this problem is to use some kind of Tikhonov regularization (Tikhonov et al. 1990). Specifically, we require the normalized autocorrelation function to be as small as possible at a given $\Delta=\Delta_{0}$, but the vector A not to be large.

This leads to a minimization problem for the functional

$$
\sum_{i=1, j=1}^{i=6, j=6} A_{i} A_{j}\left(R_{\theta_{i} \theta_{j}}\left(\Delta_{0}\right)+r \delta_{i, j}\right)-\mu\left(\sum_{i=1, j=1}^{i=6, j=6} A_{i} A_{j} R_{\theta_{i} \theta_{j}}(0)-1\right) \rightarrow \text { min, }
$$

were $r$ is the regularization parameter, which should be reasonably small, $\delta_{i j}$ is the Kronecker delta, and $\mu$ is the Lagrange multiplier for the restriction (A 3). This, in turn, can in a standard way be reduced to a linear eigenvalue problem. This method was used to obtain passive-scalar solutions with quite large and quite small streak spacing. Naturally, for linear combinations obtained in this way, the minimum of the autocorrelation function does not exactly coincide with $\Delta_{0}$, but, in practice, $\Delta_{0}$ and $\Delta_{\min }$ turn out to be close within a reasonable range of $\Delta_{0}$.

Table A1 gives the values of the parameters used and the obtained eigenvectors. Of six eigensolutions, we always choose the one giving the smallest value of $R_{\theta \theta}\left(\Delta_{0}\right)$. The cross-correlations depend on the distance to the wall. The results in the table were obtained with $R_{\theta \theta}$ being calculated at the distance $y^{+}=19.6$ from the wall. Here $I D$ is the profile identifier. The $A_{i}$ values in this table determine the profiles uniquely.

\section{Appendix B. Mathematical details of optimal perturbations independent of longitudinal coordinate}

\section{B.1. Transition to the Fourier space in spanwise coordinates}

Let us consider the solution of the variational problem (3.7) in the special case when, as in the present study, $\|.\|_{s}=\|.\|_{y}$ and the perturbations are independent of $x$. Then from the continuity equation, it follows that a cross-flow streamfunction $\psi$ can be introduced so that $v^{\prime}=-\partial \psi / \partial z, w^{\prime}=\partial \psi / \partial y$. Let $\psi=\psi_{\beta}(t, y) \mathrm{e}^{\mathrm{i} \beta z}$. Without loss of generality, $\psi_{\beta}$ can be assumed to be real-valued. Then $v^{\prime}=-\mathrm{i} \beta \psi_{\beta} \mathrm{e}^{\mathrm{i} \beta z}=\mathrm{i} v_{\beta} \mathrm{e}^{\mathrm{i} \beta z}$ and $w^{\prime}=\left(\partial \psi_{\beta} / \partial y\right) \mathrm{e}^{\mathrm{i} \beta z}=w_{\beta} \mathrm{e}^{\mathrm{i} \beta z}$ where $v_{\beta}$ and $w_{\beta}$ are also real-valued. Since with $\partial / \partial x=0$ the equation for $u^{\prime}$ involves $v^{\prime}$, but not $w^{\prime}$, it is natural to take $u^{\prime}=\mathrm{i} u_{\beta} \mathrm{e}^{\mathrm{i} \beta z}$ with real-valued $u_{\beta}$. Then the three functions $u_{\beta}(t, y), v_{\beta}(t, y)$ and $w_{\beta}(t, y)$ determine the solution which we will denote $\boldsymbol{u}_{\beta}$. 
The solution of the linearized Navier-Stokes equations is a superposition of solutions of the type $\boldsymbol{u}_{\beta}$. Solutions with different $\beta$ are orthogonal with respect to a scalar product corresponding to $\|.\|_{y}$. Therefore, solutions for each $\beta$ can be studied separately. Similarly to $\|.\|_{y}$ and $\|.\|_{c}$, we introduce $\left\|\boldsymbol{u}_{\beta}\right\|_{y, 1 D}^{2}=\left|u_{\beta}(t, y)\right|^{2}$ and $\left\|\boldsymbol{u}_{\beta}\right\|_{c, 1 D}^{2}=\int_{-1}^{+1}\left(v_{\beta}^{2}+w_{\beta}^{2}\right) \mathrm{d} y$, respectively. Naturally, the continuity equation imposes a relation between $v_{\beta}$ and $w_{\beta}$ which can be used to obtain an equivalent alternative formulation. The variational problem now consists in maximizing $u_{\beta}(t, y)$ for given $t$, $\beta$ and $y$ by varying the initial (at $t=0$ ) distribution of $v_{\beta}(y)$ and $w_{\beta}(y)$, but keeping $\left\|\boldsymbol{u}_{\beta 0}\right\|_{c, 1 D}=1$. With $\boldsymbol{u}_{\beta}=A_{1 D}(t, \beta) \boldsymbol{u}_{\beta 0}$ and scalar products $(., .)_{c, 1 D},(., .)_{y, 1 D}$ defined such that $\left\|\boldsymbol{u}_{\beta}\right\|_{c, 1 D}^{2}=\left(\boldsymbol{u}_{\beta}, \boldsymbol{u}_{\beta}\right)_{c, 1 D},\left\|\boldsymbol{u}_{\beta}\right\|_{y, 1 D}^{2}=\left(\boldsymbol{u}_{\beta}, \boldsymbol{u}_{\beta}\right)_{y, 1 D}$, the variational problem consists in maximizing

$$
F_{\beta}=\left(A_{1 D}(t, \beta) \boldsymbol{u}_{\beta 0}, A_{1 D}(t, \beta) \boldsymbol{u}_{\beta 0}\right)_{y, 1 D}+\lambda(t, y, \beta)\left(\left(\boldsymbol{u}_{\beta 0}, \boldsymbol{u}_{\beta 0}\right)_{c, 1 D}-1\right) .
$$

Here, similar to $(3.7), A_{1 D}(t, \beta)$ is a linear operator and $\lambda(t, y, \beta)$ is the Lagrange multiplier. Similar to (3.7) and (3.8) the Euler-Lagrange equation for (B 1) may be reduced to

$$
E_{t, \beta} \gamma+\lambda E_{0, \beta} \gamma=0
$$

Solving the variational problem with the functional (B1) gives $\lambda(t, y, \beta)$. The solution to the generalized optimal perturbation problem is then obtained by maximizing $\lambda(t, y, \beta)$ over all $\beta$ and all $t>0$. The value of $\beta$ gives then the most probable streak spacing $l^{+}=2 \pi R e \beta$ at this distance from the wall.

\section{B.2. Relation to a Green function}

Owing to the linearity of the problem, maximizing $u_{\beta}$ is equivalent to maximizing $\left|u_{\beta}\right|$. Since $u_{\beta}(t, y)$ is a linear functional of the initial distribution $\left(v_{\beta 0}, w_{\beta 0}\right)$, it can be represented (non-uniquely) via the Green function $\boldsymbol{G}(t, \beta, y, \eta)=$ $\left(G_{v}(t, \beta, y, \eta), G_{w}(t, \beta, y, \eta)\right)$ as

$$
u_{\beta}(t, y)=\int_{-1}^{+1}\left(G_{v}(t, \beta, y, \eta) v_{\beta 0}(\eta)+G_{w}(t, \beta, y, \eta) w_{\beta 0}(\eta)\right) \mathrm{d} \eta .
$$

The specific method of calculating $\boldsymbol{G}$ is given in Appendix C. Since (B 3) is an $L_{2}$ scalar product of $\left(G_{v}, G_{w}\right)$ and $\left(v_{\beta 0}, w_{\beta 0}\right), u_{\beta}(t, y)$ is maximized when these vectors are parallel, that is when $\left(v_{\beta 0}(\eta), w_{\beta 0}(\eta)\right)=$ const $\left(G_{v}(t, \beta, y, \eta), G_{w}(t, \beta, y, \eta)\right)$. The constant can be found from the requirement that the initial condition should have a unit initial condition norm: $\left\|\boldsymbol{u}_{\beta 0}\right\|_{c, 1 D}^{2}=\int_{-1}^{+1}\left(v_{\beta 0}^{2}+w_{\beta 0}^{2}\right) \mathrm{d} y=1$. Hence, the solution to the variational problem with functional (B 1) is

$$
\left(v_{\beta 0}(\eta), w_{\beta 0}(\eta)\right)=\frac{\boldsymbol{G}(t, \beta, y, \eta)}{\sqrt{\int_{-1}^{+1}\left(G_{v}(t, \beta, y, \eta)^{2}+G_{w}(t, \beta, y, \eta)^{2}\right) \mathrm{d} \eta}},
$$

and the Lagrange multiplier $\lambda(t, y, \beta)$ is the square of the $L_{2}$ norm of the Green function:

$$
\lambda(t, y, \beta)=\int_{-1}^{+1}\left(G_{v}(t, \beta, y, \eta)^{2}+G_{w}(t, \beta, y, \eta)^{2}\right) \mathrm{d} \eta .
$$

Note that this argument implies that the maximum is being sought over all $\left(v_{\beta 0}(\eta), w_{\beta 0}(\eta)\right)$ in $L_{2}$ and not only those satisfying the continuity equation $\beta w_{\beta}+\partial v_{\beta} / \partial y=0$. However, since $\beta G_{w}+\partial G_{v} / \partial \eta=0$ for $\boldsymbol{G}$ determined in the Appendix $C$, imposing continuity does not change the solution.

With $\|\cdot\|_{y, 1 D}$ and $\|\cdot\|_{c, 1 D}$ used, solving the eigenproblem (B2) gives the Green function and, vice versa, the Green function can be used as the solution to the 
eigenproblem. Notice that since any initial condition which is orthogonal to the Green function results in zero $u_{\beta}(t, y)$, there is only one non-zero eigenvalue. This can be used for developing an efficient numerical solver for the eigenproblem in question. It is, however, even easier to maximize (B 5) itself than to solve the eigenvalue problem.

\section{B.3. Maximizing the variation of the longitudinal velocity}

Consider a statistical ensemble of random initial conditions $\left(\hat{v}_{\beta 0}(y), \hat{w}_{\beta 0}(y)\right)$ with zero means, which corresponds to solutions $\hat{u}_{\beta}(t, y)$. As is natural for a linearized problem, the mean value of $\hat{u}_{\beta}(t, y)$ is also zero. Then, using (B 3) gives the following expression for the variance of $\hat{u}_{\beta}(t, y)$ :

$$
\begin{aligned}
\left\langle\hat{u}_{\beta}(t, y)^{2}\right\rangle= & \int_{-1}^{+1} \int_{-1}^{+1}\left(G_{v}\left(t, \beta, y, \eta_{1}\right) G_{v}\left(t, \beta, y, \eta_{2}\right)\left\langle\hat{v}_{\beta 0}\left(\eta_{1}\right) \hat{v}_{\beta 0}\left(\eta_{2}\right)\right\rangle\right. \\
& +2 G_{v}\left(t, \beta, y, \eta_{1}\right) G_{w}\left(t, \beta, y, \eta_{2}\right)\left\langle\hat{v}_{\beta 0}\left(\eta_{1}\right) \hat{w}_{\beta 0}\left(\eta_{2}\right)\right\rangle \\
& \left.+G_{w}\left(t, \beta, y, \eta_{1}\right) G_{w}\left(t, \beta, y, \eta_{2}\right)\left\langle\hat{w}_{\beta 0}\left(\eta_{1}\right) \hat{w}_{\beta 0}\left(\eta_{2}\right)\right\rangle\right) \mathrm{d} \eta_{1} \mathrm{~d} \eta_{2} .
\end{aligned}
$$

Here, we can see the similarity with the analysis of Jovanovic \& Bamieh (2001).

If we assume (rather crudely) that the characteristic correlation lengths are small and independent of $y$ and that the cross-correlation $\left\langle\hat{v}_{\beta 0} \hat{w}_{\beta 0}\right\rangle$ is negligible, then the variance reduces to

$$
\left\langle\hat{u}_{\beta}(t, y)^{2}\right\rangle=\mathrm{const} \int_{-1}^{+1}\left(G_{v}^{2}(t, \beta, y, \eta)\left\langle\hat{v}_{\beta 0}^{2}(\eta)\right\rangle+G_{w}^{2}(t, \beta, y, \eta)\left\langle\hat{w}_{\beta 0}^{2}(\eta)\right\rangle\right) \mathrm{d} \eta .
$$

On the other hand, as is easy to verify with simple algebraic transformations, using

$$
\left\|\boldsymbol{u}_{\beta}\right\|_{d, 1 D}=\int_{-1}^{+1}\left(\frac{v_{\beta}^{2}}{\left\langle\hat{v}_{\beta 0}^{2}\right\rangle}+\frac{w_{\beta}^{2}}{\left\langle\hat{w}_{\beta 0}^{2}\right\rangle}\right) \mathrm{d} y
$$

instead of $\|.\|_{c, 1 D}$, equation (B 4) becomes

$$
\left(v_{\beta 0}(\eta), w_{\beta 0}(\eta)\right)=\frac{\left(G_{v}(t, \beta, y, \eta)\left\langle\hat{v}_{\beta 0}^{2}\right\rangle, G_{w}(t, \beta, y, \eta)\left\langle\hat{w}_{\beta 0}^{2}\right\rangle\right)}{\sqrt{\int_{-1}^{+1}\left(G_{v}(t, \beta, y, \eta)^{2}\left\langle\hat{v}_{\beta 0}^{2}\right\rangle+G_{w}(t, \beta, y, \eta)^{2}\left\langle\hat{w}_{\beta 0}^{2}\right\rangle\right) \mathrm{d} \eta}},
$$

and (B 5) becomes

$$
\lambda(t, y, \beta)=\int_{-1}^{+1}\left(G_{v}^{2}(t, \beta, y, \eta)\left\langle\hat{v}_{\beta 0}^{2}(\eta)\right\rangle+G_{w}^{2}(t, \beta, y, \eta)\left\langle\hat{w}_{\beta 0}^{2}(\eta)\right\rangle\right) \mathrm{d} \eta .
$$

Assuming further that $\left(\left\langle\hat{v}_{\beta 0}^{2}\right\rangle,\left\langle\hat{w}_{\beta 0}^{2}\right\rangle\right)=\operatorname{const}\left(\left\langle v^{2}\right\rangle,\left\langle w^{2}\right\rangle\right)$ leads directly to (3.10). Note that $\left\langle v^{2}\right\rangle$ and $\left\langle w^{2}\right\rangle$ here are functions of $y$ only and they do not contain explicit information on the spanwise length scale. Note also that, in general, $\left(v_{\beta 0}, w_{\beta 0}\right)$ given by (B 8) does not satisfy the continuity equation. This is of little importance since the optimal perturbation should be interpreted as a method of investigating the properties of the linearized operator and not as a model of the Navier-Stokes solution.

\section{Appendix C. Numerical implementation details}

\section{C.1. General outline}

The specific results of the present study were obtained by the methodology which became possible with the development of computerized algebraic systems capable of exporting formulae into a highly efficient algorithmic language. Technically, the entire 
generalized optimal perturbation study was first performed with the Mathematica system, starting from the analytic transformations of the Navier-Stokes equations followed by numerical calculations and preliminary analysis of the results. Then, in order to achieve higher numerical accuracy and to allow systematic calculations of a number of cases within a reasonable time, the Mathematica notebooks were used as a prototype for the Fortran code, with large parts of the notebooks simply exported into Fortran. Physically, Mathematica notebooks are just computer files which can be interpreted by Mathematica. From the viewpoint of the researcher, Mathematica notebooks are hybrids between formula notes, an interactive algebraic manipulation system, a numerical code, and a visualization tool. A notebook can be worked with in a manner similar to working with scrap paper, but it can also be run as a code. Describing in a paper the work performed in this way presents certain difficulties. Mathematica allows us to work with analytic expressions far too long to be printed. On the other hand, the Fortran code inherits these long expressions and tends to be just a calculation by these formulae combined with the use of standard library subroutines corresponding to Mathematica commands, as, for example, the command/subroutine for calculating numerically definite integrals. Then the Fortran code does not contain much of a numerical method to talk about. Nevertheless, the method of obtaining the results should be reported, at least to an extent sufficient for independent reproduction of the results. This is the goal of this Appendix. We will limit ourselves to the calculation of the Green function $\boldsymbol{G}$, since the rest is fairly simple.

Substituting $u^{\prime}=\mathrm{i} u_{\beta}(t, y) \mathrm{e}^{i \beta z}, \quad v^{\prime}=\mathrm{i} v_{\beta}(t, y) \mathrm{e}^{i \beta z}, \quad w^{\prime}=w_{\beta}(t, y) \mathrm{e}^{i \beta z}$ and $p^{\prime}=$ i $p_{\beta}(t, y) \mathrm{e}^{i \beta z}$ into the linearized Navier-Stokes equations (3.1) with $\boldsymbol{F}=0$ gives

$$
\begin{gathered}
\frac{\partial u_{\beta}}{\partial t}+v_{\beta} U^{\prime}(y)=\frac{1}{R e}\left(\frac{\partial^{2} u_{\beta}}{\partial y^{2}}-\beta^{2} u_{\beta}\right), \\
\frac{\partial v_{\beta}}{\partial t}=-\frac{\partial p_{\beta}}{\partial y}+\frac{1}{R e}\left(\frac{\partial^{2} v_{\beta}}{\partial y^{2}}-\beta^{2} v_{\beta}\right), \\
\frac{\partial w_{\beta}}{\partial t}=\beta p_{\beta}+\frac{1}{R e}\left(\frac{\partial^{2} w_{\beta}}{\partial y^{2}}-\beta^{2} w_{\beta}\right) .
\end{gathered}
$$

The boundary conditions are $u_{\beta}=v_{\beta}=w_{\beta}=0$ at $y= \pm 1$.

The initial conditions are $t=0, u_{\beta}=0, v_{\beta}=u_{\beta 0}(y)$ and $w_{\beta}=w_{\beta 0}(y)$.

The problem is to find functions $G_{v}(t, \beta, y, \eta)$ and $G_{w}(t, \beta, y, \eta)$ such that (B 3$)$ is valid, that is, such that

$$
u_{\beta}(t, y)=\int_{-1}^{+1}\left(G_{v}(t, \beta, y, \eta) v_{\beta 0}(\eta)+G_{w}(t, \beta, y, \eta) w_{\beta 0}(\eta)\right) \mathrm{d} \eta .
$$

Introducing the streamfunction $\psi_{\beta}(t, y)$ such that $v_{\beta}=-\beta \psi_{\beta}$, and $w_{\beta}=\partial \psi_{\beta} / \partial y$ reduces (C2) and (C 3) to a single equation.

Separating variables gives solutions of the form $\exp \left(-\lambda_{k} t / R e\right) f_{k}(y)$. There is an infinite set of such solutions and corresponding values of $\lambda=\lambda_{k}, \quad k=1, \ldots, \infty$, satisfying all the boundary conditions. For each solution $v_{\beta}=-\beta \exp \left(-\lambda_{k} t / R e\right) f_{k}=$ $\exp \left(-\lambda_{k} t / R e\right) v_{k}$ and $w_{\beta}=\exp \left(-\lambda_{k} t / R e\right) f_{k}^{\prime}=\exp \left(-\lambda_{k} t / R e\right) w_{k}$.

Functions

$$
E_{m}(z)=\sin (\pi m(y+1) / 2)
$$


form a full orthonormal (in $L_{2}$ ) basis so that for each $f_{k}(y)$ there are coefficients

$$
B_{k m}=\int_{-1}^{+1} f_{k}(y) U^{\prime}(y) E_{m}(y) \mathrm{d} y
$$

such that

$$
f_{k}(y) U^{\prime}(y)=\sum_{m} B_{k m} E_{m}(y) .
$$

Substituting $v_{\beta}=\exp \left(-\lambda_{k} t / R e\right) v_{k}$ into (C1), we then obtain

$$
u_{k}=-\beta \sum_{m} B_{k m} T_{k m}(t) E_{m}(y),
$$

where

$$
T_{k m}(t)=R e \frac{\exp \left(-\left(m^{2} \pi^{2} / 4+\beta^{2}\right) t / R e\right)-\exp \left(-\lambda_{k} t / R e\right)}{\left(m^{2} \pi^{2} / 4+\beta^{2}-\lambda_{k}\right)} .
$$

As is shown in the following subsection, suitably normalized vectors $\left(v_{k}, w_{k}\right)$ form a full orthonormal basis in the sense that

$$
\int_{-1}^{+1}\left(v_{k} v_{l}+w_{k} w_{l}\right) \mathrm{d} y=\delta_{k l},
$$

where $\delta_{k l}$ is the Kronecker delta and that if

$$
\gamma_{k}=\int_{-1}^{+1}\left(v_{\beta 0} v_{k}+w_{\beta 0} w_{k}\right) \mathrm{d} y
$$

then

$$
\left(v_{\beta 0}, w_{\beta 0}\right)=\sum_{k} \gamma_{k}\left(v_{k}, w_{k}\right)
$$

Therefore,

$$
u_{\beta}=\sum_{k} \gamma_{k} u_{k}=-\beta \sum_{k, m} \gamma_{k} B_{k m} T_{k m}(t) E_{m}(y) .
$$

At this point, it is possible just to look for $\gamma_{k}, k=1, \ldots$ which maximize $u_{\beta}^{2}$ with the restriction $\sum_{k} \gamma_{k}^{2}=1$. This would lead to an eigenvalue problem, the solution to which is equivalent to calculating the Green function (see §B.2). Alternatively, substituting (C 5) into (C 7) and comparing with (B 3) gives

$$
\left(G_{v}, G_{w}\right)=-\beta \sum_{k, m} B_{k m} T_{k m}(t) E_{m}(y)\left(v_{k}(\eta), w_{k}(\eta)\right) .
$$

The entire idea of this solution is quite standard, and the specific algebraic transformations leading to the above formulae can be performed on paper without a computerized algebraic system, even though in our study we used Mathematica throughout. However, calculating $f_{k}(y)$ and $B_{k m}$ is more complicated.

\section{C.2. Eigenfunctions $f_{k}$ and coefficients $B_{k m}$}

The equation for $f_{k}$ can be obtained by simple substitutions from Navier-Stokes equations. It has the form:

$$
f_{k}^{(\mathrm{iv})}(y)-2 \beta^{2} f_{k}^{\prime \prime}(y)+\beta^{4} f_{k}(y)=\lambda_{k}\left(\beta^{2} f_{k}(y)-f_{k}^{\prime \prime}(y)\right) .
$$

The boundary conditions are $f(-1)=f^{\prime}(-1)=f(1)=f^{\prime}(-1)=0$. This is a selfadjoint positively determined eigenvalue problem. Therefore (Kamke 1983), its 
eigenfunctions are orthogonal and form a full basis in a functional space with the scalar product defined as

$$
(g, h)=\int_{-1}^{+1} g(y)\left(\beta^{2} h(y)-h^{\prime \prime}(y)\right) \mathrm{d} y .
$$

For functions satisfying the boundary conditions, integration by parts leads to

$$
(g, h)=\int_{-1}^{+1}\left(\beta^{2} g(y) h(y)+g^{\prime}(y) h^{\prime}(y)\right) \mathrm{d} y .
$$

This immediately gives (C 4)-(C 6).

Equation (C 8) can be solved analytically using Mathematica. This gives the general solution as a sum of four independent solutions each multiplied by an arbitrary coefficient. Then the requirement that the solution should satisfy four boundary conditions gives a homogeneous system of four equations for these coefficients. The eigenvalues $\lambda_{k}$ can then be found from the requirement that the determinant of this system is equal to zero. This approach leads to very complicated expressions, which are difficult to handle even with Mathematica. Another approach consists in finding a solution satisfying only three of the boundary conditions. Then the eigenvalues $\lambda_{k}$ can be sought from the fourth boundary condition. This approach does not guarantee, however, that all the eigenvalues will be found. Also, $\lambda_{k}$ has to be determined numerically, and the solutions can turn into infinity at certain values of $\lambda_{k}$, thus making the numerical calculations difficult. After some experimenting, it was found that satisfactory results could be obtained by considering two solutions of (C 8), $f_{s}(y)$ and $f_{a}(y)$, satisfying the boundary conditions

$$
\begin{aligned}
& f_{s}(-1)=f_{s}(1), \quad f_{s}^{\prime}(-1)=f_{s}^{\prime}(1)=0, \\
& f_{a}(-1)=-f_{a}(1), \quad f_{a}^{\prime}(-1)=f_{a}^{\prime}(1)=0,
\end{aligned}
$$

and having, with suitable normalization, the form

$$
\begin{aligned}
& f_{s}=\frac{\mathrm{e}^{z \beta}\left(-1+\mathrm{e}^{2 \beta}\right) \beta \cos \left(z v_{k}\right)+\mathrm{e}^{\beta}\left(1+\mathrm{e}^{2 z \beta}\right) v_{k} \sin \left(v_{k}\right)}{\mathrm{e}^{z \beta}\left(1+\mathrm{e}^{2 \beta}\right) \sqrt{\beta^{2}+v_{k}^{2}}}, \\
& f_{a}=\frac{\mathrm{e}^{\beta}\left(-1+\mathrm{e}^{2 z \beta}\right) v_{k} \cos \left(v_{k}\right)-\mathrm{e}^{z \beta}\left(1+\mathrm{e}^{2 \beta}\right) \beta \sin (z v)}{\mathrm{e}^{z \beta}\left(1+\mathrm{e}^{2 \beta}\right) \sqrt{\beta^{2}+v_{k}^{2}}},
\end{aligned}
$$

where $v_{k}=\sqrt{\lambda_{k}-\beta^{2}}$.

Each of these functions gives eigenvalues as solutions to $f_{s}(1)=0$ and $f_{a}(1)=0$. To obtain different eigenvalues, iterative numerical procedure was started from different initial approximations. For $f_{s}$, the initial approximations were $v_{k}=0.7 \pi, 1.7 \pi$, $2.7 \pi, \ldots$ and for $f_{a}$ the initial approximations were $v_{k}=1.3 \pi, 2.3 \pi, 3.3 \pi, \ldots$ To check that the obtained system was full, several test functions were expanded into a series using the obtained basis, and the sum of the series was compared to the test function itself.

To exploit the full advantage of the Mathematica's ability to perform algebraic manipulations, the coefficients $B_{k m}$ were calculated in the following way. We represent $B_{k m}$ in the form

$$
B_{k m}=\int_{-1}^{+1}\left(f_{k}(y)\left(U^{\prime}(y)-y U^{\prime}(1)\right) E_{m}(y)\right) \mathrm{d} y+U^{\prime}(1) \int_{-1}^{+1} f_{k}(y) y E_{m}(y) \mathrm{d} y .
$$


The second integral here can be calculated analytically in a general form (that is with $\lambda_{k}$ and $\beta$ as symbols in $f_{k}$ and elsewhere). The result is just a huge explicit expression. The first integral is calculated by expanding $f_{k}(y)$ in $E_{m}(y): f_{k}(y)=\sum_{m} f_{k m} E_{m}(y)$ again analytically in a general form, so that $f_{k}(y)=\sum_{m} f_{k m} E_{m}(y)$, expanding $U^{\prime}(y)-$ $y U^{\prime}(1)$ in $E_{m}(y)$ numerically so that $U^{\prime}(y)-y U^{\prime}(1)=\sum_{m} U_{m} E_{m}(y)$, and calculating

$$
\Pi_{k l m}=\int_{-1}^{1} E_{k}(z) E_{l}(z) E_{m}(z) \mathrm{d} z
$$

again analytically. Then,

$$
B_{k m}=\sum_{l, n} f_{k l} U_{n} \Pi_{l n m}+U^{\prime}(1) \int_{-1}^{+1} f_{k}(y) y E_{m}(y) \mathrm{d} y .
$$

The advantage of this approach is that all the components here are calculated only once for all values of $\beta$ and $\lambda_{k}$ either because the calculations are symbolic or because $\beta$ and $\lambda_{k}$ are not involved in the numerical part. Separating $y U^{\prime}(1)$ also greatly accelerates convergence.

Exporting large expressions to Fortran may result in a loss of accuracy owing to rounding errors. In the present study, this was checked by performing the calculations in Fortran with single and double precision and comparing the results. Rounding errors turned out to be large, but that was corrected simply by rearranging the expressions involved.

\section{REFERENCES}

Brooke, J. W. \& Hanratty, T. J. 1993 Origin of turbulence producing eddies in a channel flow. Phys. Fluids A 5, 1011-1021.

Butler, K. M. \& Farrell, B. F. 1992 Three-dimensional optimal perturbations in viscous shear flow. Phys. Fluids A 4, 1637-1650.

Butler, K. M. \& FArrell, B. F. 1993 Optimal perturbations and streak spacing in wall-bounded turbulent shear flows. Phys. Fluids A 5, 774-777.

Carpenter, P., Ali, R., Davies, C. \& Lockerby, D. 2003 A simple computational model for studying the control of viscous sublayers. In 5th Euromech Fluid Mech. Conf. Toulouse, 2428 August, p. 367.

Chernyshenko, S. I. \& Baig, M. F. 2005 Streaks and vortices in near-wall turbulence. Phil. Trans. R. Soc. Lond. A 363, 1097-1107.

Criminale, W. O., Jackson, T. L. \& Lasseigne, D. G. 1995 The initial-value problem for viscous channel flows. NASA TR 95-6. CR-195034 ICASE, Institute for Computer Applications in Science and Engineering, Mail Stop 132C, NASA Langley Research Center Hampton, VA, available at http://www.icase.edu/.

Hamilton, J. M., Kim, J. \& WalefFe, F. 1995 Regeneration mechanisms of near-wall turbulence structures. J. Fluid Mech. 232, 317-348.

Hu, Z. W., Morfey, C. L. \& Sandham, N. D. 2003 Sound radiation in turbulent channel flows. J. Fluid. Mech. 475, 269-302.

Hu, Z. W. \& Sandham, N. D. 2001 Large-domain simulations of Couette and Poiseille flow. In Proc. 2nd Intl Symp. on Turbulent and Shear Flow Phenomena. Stockholm.

JANG, P. S., Benney, D. J. \& Gran, R. L. 1986 On the origin of streamwise vortices in a turbulent boundary layer. J. Fluid Mech. 169, 109-123.

Jiménez, J., Pinelli, A. \& Uhlmann, M. 2000 Control of turbulent wall flows. School of Aeronautics - Universidad Politécnica de Madrid. ERCOFTAG Bull. 44, 7-14.

Jovanović, M. \& BamieH, B. 2001 Modeling flow statistics using the linearized Navier-Stokes equations. In Proc. of the 40th IEEE Conf. on Decision and Control, pp. 4944-4949.

KАмкE, E. 1983 Differentialgleichungen: Lösungsmethoden und Lösungen, Bd. 1: Gewöhnliche Differentialgleichungen. Teubner, Stuttgart. 
Kasagi, N., Tomita, Y. \& Kuroda, A. 1992 Direct numerical simulation of passive scalar field in a turbulent channel flow. Trans. ASME C: J. Heat Transfer 114, 598-606.

Kim, J. \& Lim, J. 2000 A linear process in wall-bounded turbulent shear flows. Phys. Fluids 12, $1885-1888$.

KIM, J. \& MoIN, P. 1989 Transport of passive scalars in a turbulent channel flow. In Turbulent Shear Flows 6 (ed. J.-C. A. André, J. Consteix, F. Durst, et al.), pp. 85-96. Springer.

LANDAHL, M. T. 1989 Boundary layer turbulence regarded as a driven linear system. Physica D 37, $11-19$.

LeE, M. J., Kim, J. \& Moin, P. 1990 Structure of turbulence at high shear rate. J. Fluid Mech. 216, $561-583$.

Nazarenko, S., Kevlahan, N. K.-R. \& Dubrulle, B. 1999 WKB theory for rapid distortion of inhomogeneous turbulence. J. Fluid Mech. 390, 325-348.

Nikitin, N. V. \& Chernyshenko, S. I. 1997 On the nature of the organized structures in turbulent near-wall flows. Fluid Dyn. 32, 18-23. (Trans. from Izvestiya Rossiiskoi Akademii Nauk, Mekhanika Zhidkosti i Gaza 1, pp. 24-30, 1997.)

Panton, R. L. (ed.) 1997 Self-Sustaining Mechanism of Wall Turbulence. Computational Mechanics Publication, Southampton.

SANDham, N. D. \& Howard, R. J. A. 1998 Direct simulation of turbulence using massively parallel computers. In Parallel Computational Fluid Dynamics. Recent Developments and Advances Using Parallel Computers (ed. D. R. Emerson, A. Ecer, J. Peraux \& P. Fox), pp. 23-32. Elsevier.

Schoppa, W. \& Hussain, F. 1998 Formation of near-wall streamwise vortices by streak instability. Tech. Rep. 98-3000. AIAA, 29th AIAA Fluid Dyn. Conf. Albuquerque, NM, USA.

Schoppa, W. \& Hussain, F. 2002 Coherent structure generation in near-wall turbulence. J. Fluid Mech. 453, 57-108.

Smith, C. R. \& MetzleR, S. P. 1983 The characteristics of low-speed streaks in the near-wall region of a turbulent boundary layer. J. Fluid Mech. 129, 27-54.

Smith, C. R. \& Walker, J. D. A. 1994 Turbulent wall layer vortices. In Fluid Vortices (ed. S. Green). Springer.

SREenivasan, K. R. 1988 A unified view of the origin and morphology of the turbulent boundary layer structure. In Proc. IUTAM Symp. on Turbulence Management and Relaminarisation (ed. H. W. Liepmann \& R. Narasimha), pp. 37-61. Springer.

StUART, J. T. 1965 The production of intense shear layers by vortex stretching and convection. NATO AGARD Rep. 514, 1-29.

Tikhonov, A. N., Goncharskit, A. V., Stepanov, V. V. \& Yagoda, A. G. 1990 Numerical Methods for Ill-Posed Problems. Nauka, Moscow. [In Russian].

Trefethen, L. N., Trefethen, A. E., Reddy, S. C. \& Driscoll, T. A. 1993 Hydrodynamics stability without eigenvalues. Science 261, 578-584.

WalefFe, F. 2003 Homotopy of exact coherent structures in plane shear flows. Phys. Fluids 6, $1517-1534$.

WalefFe, F. \& Kim, J. 1997 How streamwise rolls and streaks self-sustain in a shear flow. In Self-Sustaining Mechanism of Wall Turbulence (ed. R. L. Panton), pp. 309-331. Computational Mechanics Publication, Southampton.

WALefFe, F., Kim, J. \& Hamilton, J. 1993 On the origin of streaks in turbulent boundary layers. In Turbulent Shear Flows 8 (ed. F. Durst, R. Friedrich, B. E. Launder, F. W. Schmidt, U. Schumann \& J. Whitelaw), pp. 37-49. Springer. 